\title{
Further Investigations of Gravity Modeling on Surface-Interacting Vehicle Simulations
}

\author{
Michael M. Madden ${ }^{*}$ \\ NASA, Hampton, VA, 23681
}

\begin{abstract}
A vehicle simulation is "surface-interacting" if the state of the vehicle (position, velocity, and acceleration) relative to the surface is important. Surface-interacting simulations perform ascent, entry, descent, landing, surface travel, or atmospheric flight. The dynamics of surface-interacting simulations are influenced by the modeling of gravity. Gravity is the sum of gravitation and the centrifugal acceleration due to the world's rotation. Both components are functions of position relative to the world's center and that position for a given set of geodetic coordinates (latitude, longitude, and altitude) depends on the world model (world shape and dynamics). Thus, gravity fidelity depends on the fidelities of the gravitation model and the world model and on the interaction of the gravitation and world model. A surface-interacting simulation cannot treat the gravitation separately from the world model. This paper examines the actual performance of different pairs of world and gravitation models (or direct gravity models) on the travel of a subsonic civil transport in level flight under various starting conditions.
\end{abstract}

\section{Nomenclature and Acronyms}

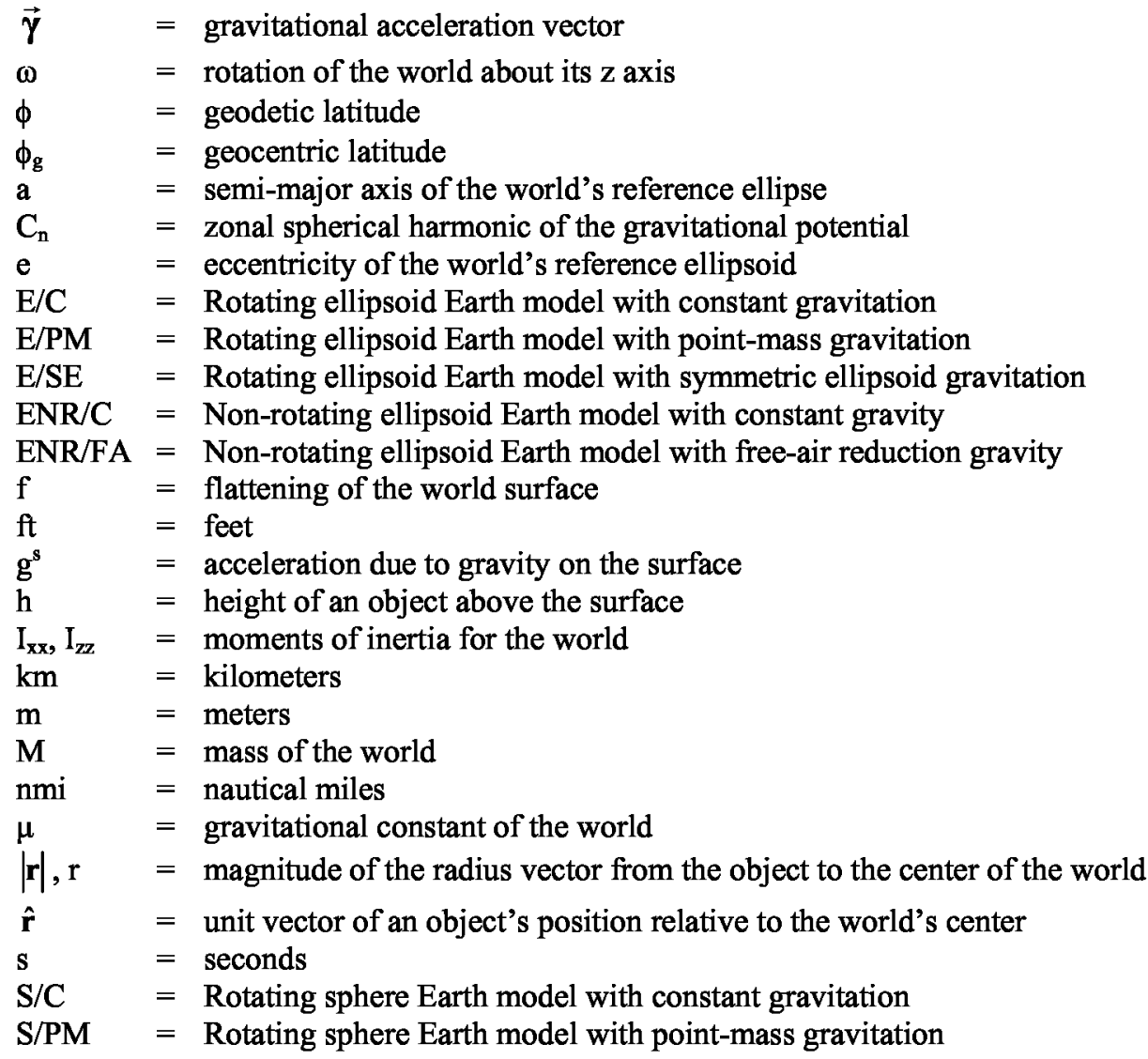

\footnotetext{
${ }^{*}$ Chief Scientist, Simulation Development and Analysis Branch, Mail Stop 125B, Senior Member AIAA.
} 
$\mathrm{S} / \mathrm{SE}=$ Rotating sphere Earth model with symmetric ellipsoid gravitation

$\mathrm{SNR} / \mathrm{C}=$ Non-rotating sphere Earth model with constant gravity

SNR/FA $=$ Non-rotating sphere Earth model with free-air reduction gravity

WGS84 $=$ World Geodetic System 1984

$\mathrm{x}, \mathrm{y}, \mathrm{z}=$ Earth-fixed Cartesian coordinates

\section{Introduction}

A erospace simulations can model worlds, such as the Earth, with differing levels of fidelity. The simulation may represent the world as a plane, a sphere, an ellipsoid, or a high-order closed surface. The world model may or may not include the rotation of the world. Likewise, the simulation can model gravity or the gravitation component, as appropriate, using models of differing fidelities; examples include constant gravity, constant gravitation, free-air reduction, point-mass gravitation, and a spherical-harmonic expansion of the geopotential. The user may select lower fidelity models based on computational limits, a need for simplified analysis, or comparison to other data. Simulation products can model the world and gravity or gravitation independently, allowing users to select any combination of world and gravity/gravitation model. The Langley Standard Real-Time Simulation in C++ (LASRS ++ $)^{1}$ presents world and gravity/gravitation models as independent user selections. Independently selecting the gravity/gravitation model and the world model does not necessarily result in a combined fidelity equivalent to the fidelity of the individual models. Some pairs produce degraded fidelity. ${ }^{2}$

This study examines the actual performance of paired world and gravity or gravitation models ("world-gravity pairs") in a simulation using the Earth. The choice of world-gravity pairs is based on prior analytical work in Reference 2; the pairs are described in section II.C. The selected world-gravity pairs are applied to a simulation of a large subsonic aircraft in level flight that is initialized to various locations and track angles. Performance of the worldgravity pairs is measured as the deviation of the aircraft's trajectory from the trajectory produced by a reference world-gravity pair. The reference world-gravity pair models a rotating WGS- $84^{3}$ ellipsoid of the Earth whose density is symmetric about the polar axis and the equator. The simulation scenarios examined here expand the work in Reference 4, which presented the performance of the same world-gravity pairs using the same aircraft simulation on a route that started at the equator and flew north. This paper presents results from eight additional scenarios. The scenarios are described in section III; the resulting aircraft trajectories are discussed in section IV. The next section provides a brief overview of the world and gravity/gravitation models; more detailed treatment of these models is found in Reference 2.

\section{The Models}

\section{A. World Models}

World models simulate the motion of the world and provide an approximation of its shape. For the purposes of this investigation, a world is a body whose shape is roughly spherical under the influence of its self-gravity. This study examines the sphere and ellipsoid shape models that are commonly used to model the Earth. This study does not address modeling of the Earth as a plane, i.e. the "flat Earth" model. The modeling of gravity or gravitation becomes important only as one travels large distances over the Earth, and "flat Earth" models are valid only under comparatively small distances. The sphere model has a constant radius and is a good approximation for bodies whose self-gravity defines its shape. Choices for the radius include: the equatorial radius, the average of the equatorial and polar radii, the mean radius, the radius of a sphere with surface area equal to the reference ellipsoid, and the radius of a sphere with volume equal to the reference ellipsoid. Because it minimizes error in the mean surface gravity, the radius of a sphere with equal surface area to the ellipsoid was chosen to model the Earth. ${ }^{2}$ The ellipsoid model describes the Earth as an ellipse of revolution about its semi-minor axis (i.e. an oblate spheroid). The ellipsoid is defined as an equipotential surface of the potential of gravity. ${ }^{3}$ This investigation uses the ellipsoid of the World Geodetic System 1984 (WGS84). ${ }^{3}$ WGS84 characterizes the ellipsoid using four parameters: the semi-major axis (a), the Earth's gravitational constant $(\mu)$, the flattening (f), and the angular velocity $(\omega)$ of the Earth.

Vehicles in surface interacting simulations are bound to the world by gravitation. Thus, only the world's motion relative to its center of mass, i.e. its rotation, is relevant to surface-interacting dynamics. The Earth has a dominant rotation about one axis and that axis is used as the z-axis in Earth-fixed, Earth-centered Cartesian coordinate systems. Both the sphere and ellipsoid can model the rotation of the world, and modeling rotation will increase the overall fidelity of those models. These rotating world models use a constant rotation of $7.292115 \times 10^{-5}$ for the Earth. ${ }^{3}$ But, one can also set the rotation to zero for simplicity. Disabling rotation removes the centrifugal accelera- 
tion and Coriolis acceleration terms in the acceleration equation for a vehicle when observed in an Earth-fixed frame. The centrifugal acceleration term is a function of vehicle position. Its mean value on the surface of the Earth is $0.23 \%$ of gravity. The Coriolis acceleration term is proportional to the object's velocity and remains small only for low speed vehicles (e.g. subsonic). Thus, non-rotating worlds can be appropriate for simulations of low-speed vehicles whose travel typically is limited to the world's surface. For the simulation scenarios used in this investigation, the magnitude of the Coriolis acceleration term is limited to $0.31 \%$ of mean gravity.

Rotation also directly impacts how gravity is modeled. Gravity is the combination of gravitation and centrifugal acceleration. ${ }^{5}$ If rotation is turned off, centrifugal acceleration is not modeled, and a surface interacting simulation must model gravity directly in order to reproduce the free fall of objects as observed from the Earth surface. Modeling gravitation without modeling rotation will produce slightly larger free fall at locations away from the poles (where the centrifugal acceleration is zero). If rotation is modeled, then the simulation should not use a gravity model; doing so will exaggerate the affect of the centrifugal acceleration. Only gravitation models should be selected when rotation is modeled.

\section{B. Gravity and Gravitation Models}

This study examines two gravity models and three gravitation models. The gravity models are paired with nonrotating world models; the gravitation models are paired with rotating world models. The gravity models are constant gravity and the free air reduction. The gravitation models are constant gravitation, point-mass gravitation, and gravitation of a symmetric ellipsoid.

\section{Constant Gravity and Gravitation}

The constant gravity and gravitation models use one value for gravity or gravitation regardless of the vehicle's location. Gravity acts along the surface normal and gravitation acts along the geocentric radius between the vehicle and the center of the Earth. ${ }^{\dagger}$ The gravity constant selected for this model is standard gravity, $9.80665 \mathrm{~m} / \mathrm{s}^{2}(32.174$ $\mathrm{ft} / \mathrm{s}^{2}$ ). Standard gravity is a standard unit of acceleration ("g") and not a physical property of the Earth. However, its error profile against the WGS84 gravity formula shows improved fidelity over the mean gravity (which is a physical property). ${ }^{2}$ The gravitation constant selected for this model is the mean gravitation, $9.82023 \mathrm{~m} / \mathrm{s}^{2}\left(32.219 \mathrm{ft} / \mathrm{s}^{2}\right)$.

\section{Free Air Reduction}

The free air reduction is a linear approximation for the vertical gradient of gravity in the neighborhood of the reference geoid; the linear change in gravity with height is approximately $3.086 \times 10^{-6} \mathrm{~s}^{-2}{ }^{6}$. Its gravity equation is:

$$
g=g^{s}+3.086 \times 10^{-6} h
$$

where $\mathrm{g}^{\mathrm{s}}$ is the reference gravity on the surface and $\mathrm{h}$ is the altitude. Standard gravity is used for $\mathrm{g}^{\mathrm{s}}$.

\section{Point-Mass Gravitation}

The point-mass gravitation model applies Newton's universal law of gravitation to a spherically symmetric mass. A spherically symmetric mass can be treated as if all of its mass where located at its center, i.e. a point mass. Equation 2 is the formula for point-mass gravitation:

$$
\vec{\gamma}=-\frac{\mu}{|\mathbf{r}|^{2}} \hat{\mathbf{r}}
$$

\section{Symmetric Ellipsoid Gravitation}

The Earth is not a spherically symmetric mass. The Earth bulges at the equator as a consequence of its rotation. The density of the Earth also varies from location to location. The variability of the Earth's mass is modeled by expressing the Earth's gravitational potential as a spherical harmonic expansion. The symmetric ellipsoid gravitation model uses a simplified spherical harmonic expansion that assumes the Earth's mass is symmetric about the pole and equator. The gravitation vector is the gradient of that harmonic expansion. Equations 3 and 4 express the resulting gravitation equations to a degree of four:

\footnotetext{
${ }^{\dagger}$ Constant gravitation better predicts surface gravity by a small fraction when applied to the geodetic unit vector. ${ }^{2}$ However, constant gravitation produces lower trajectory errors when applied to the geocentric unit vector. ${ }^{4}$
} 


$$
\begin{aligned}
& \gamma_{r}=-\frac{\mu}{r^{2}}\left[1+\frac{3}{2}\left(\frac{a}{r}\right)^{2} C_{2}\left(3 \sin ^{2} \phi_{g}-1\right)+\frac{5}{8}\left(\frac{a}{r}\right)^{4} C_{4}\left(35 \sin ^{4} \phi_{g}-30 \sin ^{2} \phi_{g}+3\right)\right. \\
& \gamma_{\phi_{g}}=\cos \phi_{g} \sin \phi_{g} \frac{\mu}{r^{2}}\left(\frac{a}{r}\right)^{2}\left[3 C_{2}+\frac{1}{2}\left(\frac{a}{r}\right)^{2} C_{4}\left(35 \sin ^{2} \phi_{g}-15\right)\right.
\end{aligned}
$$

where,

$$
\begin{array}{ll}
\gamma_{r}, \gamma_{\phi_{g}} & \text { are the components of gravity along the geocentric radius and latitudinal (geocentric) directions } \\
\phi_{\mathrm{g}} & \text { is the geocentric latitude } \\
\mathrm{r} & \text { is the geocentric radius } \\
\mu & \text { is the universal gravitational constant } \\
\mathrm{a} & \text { is the semi-major axis of the reference ellipsoid } \\
\mathrm{C}_{\mathrm{n}} & \text { are the even zonal harmonic coefficients of degree } \mathrm{n}
\end{array}
$$

The simulation in this study uses equations expanded to degree $(n)=8$ because the resulting equations match WGS84 published gravitation values to ten significant digits. ${ }^{2}$ For a symmetric ellipsoid mass, the even zonal harmonic coefficients ( $C_{n}$ where $n$ is even) are functions of the world's mass (M), equatorial radius (a), and moments of inertia (Ixx, Izz) as shown in Equation 5:, 7

$$
C_{2}=\frac{I_{z z}-I_{x x}}{M a^{2}}, C_{2 \times n}=(-1)^{n} \frac{3 e^{2 \times n}}{(2 \times n+1)(2 \times n+3)}\left(1-n-5 \times n \frac{C_{2}}{e^{2}}\right)
$$

where e is the eccentricity of the Earth. The simulation used the WGS 84 value of $\mathrm{C}_{2}$ without normalization, $-0.00108262982131 .^{3}$

\section{Paring World and Gravity/Gravitation Models}

The world and gravity/gravitation models can be combined to produce ten pairs. This is less than the number of possible permutations because some permutations are eliminated due to incompatible physics. Gravity models are paired only with non-rotating world models. Likewise, gravitation models are paired only with rotating world models. The remaining ten pairs are:

- Rotating ellipsoid Earth with symmetric ellipsoid gravitation (E/SE)

- Rotating ellipsoid Earth with point-mass gravitation (E/PM)

- Rotating ellipsoid Earth with constant gravitation (E/C)

- Rotating sphere Earth with symmetric ellipsoid gravitation (S/SE)

- Rotating sphere Earth with point mass gravitation (S/PM)

- Rotating sphere Earth with constant gravitation (S/C)

- Non-rotating ellipsoid Earth with free air reduction (ENR/FA)

- Non-rotating ellipsoid Earth with constant gravity (ENR/C)

- Non-rotating sphere Earth with free air reduction (SNR/FA)

- Non-rotating sphere Earth with constant gravity (SNR/C)

Because all pairs produce gravity to a surface observer, the pairs will be called world-gravity pairs. The first pair (E/SE) matches the theoretical surface gravity of a rotating Earth in hydrostatic equilibrium and is used as the reference model against which the trajectory performance of the other pairs is compared. This paper uses the following terms to refer to related groups of gravity models:

- reduced-fidelity, world-gravity pairs = E/PM, E/C, S/SE, S/PM, S/C, ENR/FA, ENR/C, SNR/FA, and SNR/C

- rotating world-gravity pairs = E/PM, E/C, S/SE, S/PM, and S/C (excludes E/SE unless otherwise noted)

- non-rotating world-gravity pairs = ENR/FA, ENR/C, SNR/FA, and SNR/C

\footnotetext{
${ }^{\ddagger}$ Geocentric latitude is the angle between the equatorial plane and the radius from the center of the world to a point. ${ }^{3}$

$\S$ The symmetric ellipsoid gravitation model includes physical properties of the world model, like eccentricity, as constants. Because the sphere's physical properties would reduce the symmetric ellipsoid gravitation model to a point-mass model, the physical properties of the WGS84 reference ellipsoid are substituted when pairing this gravitation model with the sphere. As a result, the formula for the symmetric ellipsoid gravitation model is identical between the sphere and ellipsoid world models.
} 
- ellipsoid world-gravity pairs = E/PM, E/C, ENR/FA, and ENR/C (excludes E/SE unless otherwise noted)

- sphere world-gravity pairs = S/SE, S/PM, S/C, SNR/FA, and SNR/C

\section{Method and Prior Work}

This study uses eight routes to evaluate the surface-relative dynamics of a large subsonic aircraft under each world-gravity pair. The aircraft flies without an active control system ("open-loop") to freely react to changes in gravity. Table 1 presents the initial conditions for each route. The new routes are derived from the north route in Reference 4; the new routes differ in starting location and track angle of the aircraft. The initial speed, altitude, and world relative acceleration remain the same for each route. Initial orientation differs only in pitch to balance lift against the force of gravity. Initial angular velocity differs only in pitch rate, calculated to maintain orientation as the aircraft flies over the curved surface of the Earth. The initial velocity is defined as a world-relative value and has a speed of $\sim 675 \mathrm{ft} / \mathrm{s}$ ( 280 knots indicated air speed). At this speed, the aircraft could travel from the equator to pole in $\sim 13.5$ hours. The simulation elapsed time is set to 12 hours during which the aircraft flies approximately 4800 nautical miles. The simulation integrates the vehicle state at a $50 \mathrm{~Hz}$ rate, which is the verified and validated rate for the aircraft model.

Table 1 Initial Conditions for Simulated Routes

\begin{tabular}{|c|c|c|c|c|c|}
\hline \multirow{2}{*}{\multicolumn{2}{|c|}{ (20) }} & $\begin{array}{l}\text { South from } 80^{\circ} \\
\text { Latitude }\end{array}$ & West from Equator & $\begin{array}{l}\text { West from } 35^{\circ} \\
\text { Latitude }\end{array}$ & $\begin{array}{l}\text { East from } 80^{\circ} \\
\text { Latitude }\end{array}$ \\
\hline & geodetic latitude (deg) & 79.90476493 & 0 & 35.38232148 & 79.90476493 \\
\hline \multicolumn{2}{|l|}{ Longitude (deg) } & 34.65355218 & 32.45 & 32.45 & 34.65355218 \\
\hline \multicolumn{2}{|l|}{ altitude $(\mathrm{ft})$} & \multicolumn{4}{|c|}{$24278.2152231 \mathrm{ft}$} \\
\hline \multicolumn{2}{|c|}{ world-relative velocity (ft/s) } & {$[-674.8311157,0,0]$} & {$[0,-674.8311157,0]$} & {$[0,-674.8311157,0]$} & {$[0,674.8311157,0]$} \\
\hline \multirow{3}{*}{$\begin{array}{l}\text { world-relative } \\
\text { orientation (deg) }\end{array}$} & $\phi$ & $\sim^{\mathrm{a}}$ & $\sim^{\mathrm{a}}$ & $\sim 0^{\mathrm{a}}$ & $\sim^{\mathrm{a}}$ \\
\hline & $\theta$ & 2.3757 to $2.3974^{b}$ & 2.3708 to $2.3890^{b}$ & 2.3757 to $2.3913^{b}$ & 2.3757 to $2.3958^{b}$ \\
\hline & $\psi$ & $-0^{a}$ & $0^{a}$ & $\sim 0^{a}$ & $\sim 0^{\mathrm{a}}$ \\
\hline \multirow{3}{*}{$\begin{array}{l}\text { world-relative angular } \\
\text { velocity in body } \\
\text { coordinates (deg/sec) }\end{array}$} & $\mathbf{P}$ & $-0^{\mathrm{a}}$ & $-0^{\mathrm{a}}$ & $-0^{\mathrm{a}}$ & $\sim^{\mathrm{a}}$ \\
\hline & Q & $\begin{array}{r}-0.001848 \text { to } \\
-0.001840^{b} \\
\end{array}$ & $\begin{array}{r}-0.001848 \text { to } \\
-0.001846^{b} \\
\end{array}$ & $\begin{array}{r}-0.001848 \text { to } \\
-0.001844^{b} \\
\end{array}$ & $\begin{array}{l}-0.001848 \text { to } \\
-0.001840^{b}\end{array}$ \\
\hline & $\mathbf{R}$ & $\sim 0^{\mathrm{a}}$ & $-0^{a}$ & $-0^{\mathrm{a}}$ & $\sim 0^{a}$ \\
\hline \multirow{2}{*}{\multicolumn{2}{|c|}{ elapsed time }} & \multicolumn{4}{|c|}{12 hours } \\
\hline & & $\begin{array}{c}\text { North-East From } \\
\text { Equator }\end{array}$ & $\begin{array}{c}\text { North-West From } \\
\text { Equator }\end{array}$ & $\begin{array}{l}20^{\circ} \text { Track from } \\
\text { Equator }\end{array}$ & $\begin{array}{c}340^{\circ} \text { Track from } \\
\text { Equator }\end{array}$ \\
\hline \multicolumn{2}{|l|}{ geodetic latitude (deg) } & \multicolumn{4}{|c|}{0 degrees } \\
\hline \multicolumn{2}{|l|}{ Longitude (deg) } & \multirow{2}{*}{\multicolumn{4}{|c|}{$\begin{array}{c}32.45 \text { degrees } \\
24278.2152231 \mathrm{ft}\end{array}$}} \\
\hline \multirow{2}{*}{\multicolumn{2}{|c|}{$\begin{array}{l}\text { altitude }(\mathrm{ft}) \\
\text { world-relative velocity }(\mathrm{ft} / \mathrm{s})\end{array}$}} & & & & \\
\hline & & {$[477.18,477.18,0]$} & {$[477.18,-477.18,0]$} & {$[637.16,222.32,0]$} & {$[637.16,-222.32,0]$} \\
\hline \multirow{3}{*}{$\begin{array}{l}\text { world-relative } \\
\text { orientation (deg) }\end{array}$} & $\phi$ & $\sim 0^{a}$ & $-0^{a}$ & $\sim 0^{\mathrm{a}}$ & $0^{a}$ \\
\hline & $\theta$ & 2.3501 to $2.3850^{b}$ & 2.3672 to $2.3854^{b}$ & 2.3546 to $2.3850^{b}$ & 2.3626 to $2.3850^{b}$ \\
\hline & $\psi$ & $-0^{\mathrm{a}}$ & $-0^{a}$ & $-0^{a}$ & $-0^{a}$ \\
\hline \multirow{3}{*}{$\begin{array}{l}\text { world-relative angular } \\
\text { velocity in body } \\
\text { coordinates }(\mathrm{deg} / \mathrm{sec})\end{array}$} & $\mathbf{P}$ & $-0^{a}$ & $-0^{a}$ & $-0^{a}$ & $-0^{a}$ \\
\hline & $\mathrm{Q}$ & $\begin{array}{l}-0.001852 \text { to } \\
-0.001848^{b} \\
\end{array}$ & $\begin{array}{r}-0.001852 \text { to } \\
-0.001848^{b} \\
\end{array}$ & $\begin{array}{r}-0.001857 \text { to } \\
-0.001848^{b} \\
\end{array}$ & $\begin{array}{l}-0.001857 \text { to } \\
-0.001848^{b}\end{array}$ \\
\hline & $\mathrm{R}$ & $\sim 0^{\mathrm{a}}$ & $-0^{a}$ & $\sim 0^{a}$ & $\sim 0^{\mathrm{a}}$ \\
\hline \multicolumn{2}{|l|}{ elapsed time } & \multicolumn{4}{|c|}{12 hours } \\
\hline
\end{tabular}

The South from $80^{\circ}$ Latitude route is the north route of Reference 4 in reverse. It starts where the E/SE trajectory in the north route ended and flies due south. The south route examines whether the performance trends presented in Reference 4 continue to hold when the route is reversed. The West from Equator route starts at the same location as the north route in Reference 4 but flies due west. The equatorial radius differs between the ellipsoid and sphere world models and that difference will influence the results for this route. The ellipsoid and sphere surface have the same geocentric radius at $35.38232148^{\circ}$ latitude. Therefore, the west route was replicated at that latitude; this is the 
West from $35^{\circ}$ Latitude route. However, a free-flying aircraft will not maintain latitude outside of the equator; it will turn south to fly a great circle route and return to the region where the geocentric radius of the surface is smaller on the sphere than on the ellipsoid. The East from $80^{\circ}$ Latitude route causes the aircraft to spend more time north of $35^{\circ}$ latitude where the surface lies at a greater geocentric radius on the sphere than on the ellipsoid. The NorthEast from Equator scenarios looks at a route with a $45^{\circ}$ track and the North-West from Equator looks at the mirror route $\left(-45^{\circ}\right.$ track). These latter two scenarios, though, spend a majority of time below $35^{\circ}$ latitude where the geocentric radius of the sphere surface is smaller than that of the ellipsoid. The $20^{\circ}$ Track from Equator and $340^{\circ}$ Track from Equator routes provide almost equal time above and below $35^{\circ}$ latitude in an attempt to reduce the position errors due to differences in the geocentric radius of the surface between the sphere and ellipsoid.

\section{A. Prior Work}

References 2 and 4 lay the ground work for this study. In reference 2, the fidelity of the world-gravity pairs in reproducing gravity at the surface was analyzed. The analysis included a finding that the rotating world-gravity pairs (except E/SE) produce a residual component of gravity that is tangent to the surface. For the E/PM and E/C world-gravity pairs, the surface mean of this residual tangent gravity was $0.01073 \mathrm{~m} / \mathrm{s}^{2}$ toward the pole. The S/PM and $S / C$ world-gravity pairs produce slightly larger mean tangent gravity of $-0.01129 \mathrm{~m} / \mathrm{s}^{2}$, directed in the opposite direction toward the equator. The S/SE word-gravity pair produced the largest mean tangent gravity of -0.02194 $\mathrm{m} / \mathrm{s}^{2}$, also directed toward the equator. In Reference 4 , this residual tangent gravity was identified as the primary contributor to position error in the rotating world-gravity pairs for the subsonic aircraft scenario. The primary contributor of position error in the non-rotating world pairs was missing Coriolis acceleration. This identification was made possible because some of the ellipsoid world-gravity pairs differ from the reference E/SE pair mainly by these contributors. The E/PM and $\mathrm{E} / \mathrm{C}$ world-gravity pairs isolate the effects of residual tangent gravity. The ENR/FA and ENR/C world-gravity pairs isolate the effects of missing Coriolis acceleration. Reduced surface fidelity of the sphere model ("surface modeling error") was assumed to contribute the remaining difference in position error between sphere and ellipsoid world-gravity pairs using the same gravity or gravitation model. But, these differences were small compared to the total error. Also, there was no world-gravity pair to isolate and quantify the effect of reduced surface fidelity on position error. Therefore, there was no quantifiable link between reduced surface fidelity and the position error differences among sphere and ellipsoid world-gravity pairs. Upon comparing the position errors in the subsonic aircraft scenario, the position errors of trajectories created with non-rotating world-gravity pairs were almost half the position error for trajectories created with rotating world-gravity pairs. The residual tangent gravity had a greater impact on trajectories than the missing Coriolis acceleration. The order of increasing position modeling error (and reduced trajectory fidelity) was: ENR/FA \& ENR/C, SNR/FA \& SNR/C, E/C, E/PM, $\mathrm{S} / \mathrm{C}, \mathrm{S} / \mathrm{PM}$, and S/SE.

Based on this prior work, the analysis in this paper will also explain the position errors of the trajectories for each world-gravity pair using the three contributors identified in Reference 4: residual tangent gravity, missing Coriolis acceleration, and reduced surface fidelity. For each of the eight routes, the paper will also compare the trends in position error against the results of Reference 4. However, the results for the S/SE world-gravity pair are based on a new approach to applying the symmetric ellipsoid gravitation model on the sphere world model. The next section describes the new approach.

\section{B. Revisiting Application of the Symmetric Ellipsoid Gravitation Model on a Sphere World}

The symmetric ellipsoid gravitation model computes the gravitation vector as a function of geocentric position. On the sphere, the geodetic and geocentric positions of the aircraft are identical. References 2 and 4 took advantage of this identity and passed the geodetic position of the vehicle on the sphere directly into the symmetric ellipsoid gravitation equation. This approach decouples the gravitation field from the surface modeling of the world. The gravitation field described by the model is the same for both the sphere and ellipsoid world. A vehicle would experience the same gravitation vector at the same geocentric location regardless of whether a sphere or ellipsoid lies beneath. However, the aircraft experiences different gravitation vectors along the same surface relative route between the sphere and ellipsoid world models because the geocentric position of the vehicle differs between the sphere and ellipsoid at the same geodetic location. This approach establishes a field that is constant between ellipsoid and sphere in an Earth-Centered, Earth-Fixed frame; thus, this approach will be referred to as the ECEF Gravitation Field method. In Reference 4, this approach to the S/SE world-gravity pair produces the greatest position error among all the world-gravity pairs in the subsonic aircraft scenario because the ECEF gravitation field produces a mean tangent gravity that is double the mean tangent gravity of other rotating world-gravity pairs. 
One alternative approach is to establish identical gravitation fields between the sphere and ellipsoid for the same geodetic coordinates. In other words, the gravitation vector at a given latitude, longitude, and altitude is the same on both the sphere and the ellipsoid. This is accomplished by treating the latitude, longitude, and altitude on the sphere as if they were geodetic coordinates on the ellipsoid and computing the equivalent geocentric location on the ellipsoid. That computed geocentric location is passed to the symmetric ellipsoid gravitation equations to calculate the gravitation vector. Though this approach outputs identical gravitation vectors for the same geodetic coordinates on the sphere and ellipsoid, the centrifugal acceleration component of gravity remains a function of the real geocentric location of the vehicle. This approach will be called the Surface-Fixed Gravitation Field. Table 2 shows the maximum position error for the two methods of computing the S/SE gravitation vector. The table includes the north route from Reference 4 plus the eight routes in Table 1.

Table 2 Comparison of Approaches for Computing Gravitation with S/SE World-Gravity Pair

\begin{tabular}{|l|c|c|c|c|c|c|c|c|c|}
\hline \multirow{3}{*}{ Approach } & \multicolumn{10}{|c|}{\begin{tabular}{c} 
Maximum Position Error (nmi) \\
\cline { 2 - 10 }
\end{tabular}} & $\begin{array}{c}\text { North } \\
\text { from } \\
\text { Equator }\end{array}$ & $\begin{array}{c}\text { South } \\
\text { from } \\
80^{\circ} \\
\text { Latitude }\end{array}$ & $\begin{array}{c}\text { West } \\
\text { from } \\
\text { Equator }\end{array}$ & $\begin{array}{c}\text { West } \\
\text { from } \\
35^{\circ} \\
\text { Latitude }\end{array}$ & $\begin{array}{c}\text { East } \\
\text { from } \\
80^{\circ} \\
\text { Latitude }\end{array}$ & $\begin{array}{c}\text { North- } \\
\text { East } \\
\text { From } \\
\text { Equator }\end{array}$ & $\begin{array}{c}\text { North- } \\
\text { West } \\
\text { from } \\
\text { Equator }\end{array}$ & $\begin{array}{c}20^{\circ} \\
\text { Track } \\
\text { from } \\
\text { Equator }\end{array}$ & $\begin{array}{c}340^{\circ} \\
\text { Track } \\
\text { from } \\
\text { Equator }\end{array}$ \\
\hline $\begin{array}{l}\text { ECEF } \\
\text { Gravitation } \\
\text { field }\end{array}$ & 97.9536 & 53.2610 & 5.3641 & 46.3576 & 94.7949 & 50.7325 & 51.5536 & 86.6825 & 87.3269 \\
\hline $\begin{array}{l}\text { Surface-fixed } \\
\text { Gravitation } \\
\text { field }\end{array}$ & 10.1659 & 5.6805 & 5.3633 & 4.6769 & 6.9830 & 12.1169 & 12.1011 & 8.7740 & 8.7742 \\
\hline
\end{tabular}

In all routes except West from Equator, the surface-fixed gravitation field outperforms the ECEF gravitation field by up to an order of magnitude. As stated earlier, the residual tangent gravity is the main contributor to position error in the S/SE world-gravity. Along the equator, the residual tangential gravity is zero under both approaches; therefore, identical position errors in the West from Equator route are expected. Based on position error differences in the remaining routes, the residual tangent gravity produced by the surface-fixed gravitation field is expected to at least an order of magnitude less than the ECEF gravitation field. For the ECEF gravitation field, the mean tangent gravity is $0.02194 \mathrm{~m} / \mathrm{s}^{2}\left(0.0712 \mathrm{ft} / \mathrm{s}^{2}\right)$ toward the equator. The surface-fixed gravitation field contributes to a meant tangent gravity of $0.00007 \mathrm{~m} / \mathrm{s}^{2}\left(0.00023 \mathrm{ft} / \mathrm{s}^{2}\right){ }^{* *}$ It is nearly zero. This occurs because, in the surface-fixed gravitation field, the radial component of the gravity vector points in the direction of the computed geocentric latitude, not the actual geocentric latitude. The computed geocentric latitude will be smaller or equal to the actual geocentric latitude. Therefore, the radial component of gravity is deflected away from the surface normal toward the pole and counteracts the tangent component of centrifugal acceleration that points toward the equator. In achieving this near elimination of tangent gravity, however, the surface-fixed gravitation field sacrifices fidelity in the normal component of gravity. The mean error of normal gravity is $0.03292 \mathrm{~m} / \mathrm{s}^{2}\left(0.1080 \mathrm{ft} / \mathrm{s}^{2}\right)$ and nearly doubles that of the ECEF gravitation field whose mean error is $0.01692 \mathrm{~m} / \mathrm{s}^{2}\left(0.0555 \mathrm{ft} / \mathrm{s}^{2}\right){ }^{2}$ Nevertheless, the increased error in mean gravity has a lesser affect on position errors because the aircraft is initialized to offset normal gravity with aerodynamic lift. The elimination of tangent gravity provides another benefit. The remaining major difference between the S/SE and reference E/SE world-gravity pairs is the reduced surface fidelity of S/SE. Therefore, the S/SE worldgravity pair isolates the effect of reduced surface fidelity on position errors. The S/SE world-gravity pair now fills the gap in isolating each of the contributors to position error. Because of this benefit and the reduced position errors, the S/SE world-gravity pair will use the surface-fixed gravitation field.

\section{Results}

Figure 1 through Figure 32 present the results of the simulations. The figures depict the geodetic latitude and longitude of the resulting vehicle trajectories. A plot from start-to-finish and a plot of travel in the last hour are both provided. (Note that the latitude or longitude travel produced with one or more related world-gravity pairs may be nearly identical on a given route; therefore, some plot lines may be hidden behind others.) Altitude differences also appear among the trajectories (errors of up to 850 feet in some cases). However, those differences are tiny in comparison to the total position error and are not necessary to explain the trajectory differences among world-gravity

\footnotetext{
** See Reference 2 for more details on computing the mean tangent gravity and the mean error of normal gravity.
} 
pairs. Therefore, altitude data is not presented or discussed. The simulation results are further reduced to a series of position errors. To compute the position error, the geodetic coordinates of each run are transformed into geocentric position vector using equations 4-14 and 4-15 in Reference 2 . These equations relocate the geodetic coordinates onto the ellipsoid Earth so that geodetic coordinates of all trajectories are referenced to the same shape. The position vector for the trajectory of each world-gravity pair is subtracted by the position vector for the trajectory of the reference world-gravity pair, E/SE. The result is the position error relative to the reference pair (E/SE). For each worldgravity pair, Table 4 through Table 11 present the maximum magnitude of the position error and the magnitude of the position error at the end of the trajectory. For comparison, Table 3 also provides the position errors for the north route of Reference 4.

Table 4 South from $80^{\circ}$

Table 3 North from Equator

\begin{tabular}{|l|r|r|}
\hline $\begin{array}{l}\text { World- } \\
\text { gravity } \\
\text { pair }\end{array}$ & $\begin{array}{c}\text { Max } \\
\text { distance } \\
\text { error (nmi) }\end{array}$ & $\begin{array}{c}\text { Final } \\
\text { distance } \\
\text { error (nmi) }\end{array}$ \\
\hline E/PM & 50.7700 & 50.7700 \\
\hline E/C & 50.6162 & 50.6162 \\
\hline S/SE & 10.1659 & 5.5504 \\
\hline S/PM & 57.6159 & 57.6159 \\
\hline S/C & 57.4480 & 57.4480 \\
\hline ENR/FA & 23.3144 & 23.3144 \\
\hline ENR/C & 23.3144 & 23.3144 \\
\hline SNR/FA & 23.9506 & 23.9506 \\
\hline SNR/C & 23.9506 & 23.9506 \\
\hline
\end{tabular}

Table 6 West from $35^{\circ}$ Latitude

\begin{tabular}{|l|r|r|}
\hline $\begin{array}{l}\text { World- } \\
\text { gravity } \\
\text { pair }\end{array}$ & $\begin{array}{c}\text { Max } \\
\text { distance } \\
\text { error (nmi) }\end{array}$ & $\begin{array}{c}\text { Final } \\
\text { distance } \\
\text { error (nmi) }\end{array}$ \\
\hline E/PM & 19.9541 & 19.9541 \\
\hline E/C & 19.8991 & 19.8991 \\
\hline S/SE & 4.6769 & 4.2902 \\
\hline S/PM & 25.8655 & 25.8655 \\
\hline S/C & 25.8655 & 25.8655 \\
\hline ENR/FA & 13.5730 & 13.2998 \\
\hline ENR/C & 13.5812 & 13.3105 \\
\hline SNR/FA & 15.9410 & 15.9410 \\
\hline SNR/C & 15.9410 & 15.9410 \\
\hline
\end{tabular}

Table 9 North West from Equator

\begin{tabular}{|l|r|r|}
\hline $\begin{array}{l}\text { World- } \\
\text { gravity } \\
\text { pair }\end{array}$ & $\begin{array}{c}\text { Equator } \\
\text { Max } \\
\text { distance } \\
\text { error (nmi) }\end{array}$ & $\begin{array}{c}\text { Final } \\
\text { distance } \\
\text { error (nmi) }\end{array}$ \\
\hline E/PM & 27.6588 & 27.6588 \\
\hline E/C & 27.5977 & 27.5977 \\
\hline S/SE & 12.1011 & 12.1011 \\
\hline S/PM & 26.3735 & 26.3735 \\
\hline S/C & 26.2851 & 26.2851 \\
\hline ENR/FA & 15.6889 & 15.6889 \\
\hline ENR/C & 15.6677 & 15.6677 \\
\hline SNR/FA & 6.4093 & 3.6284 \\
\hline SNR/C & 6.4093 & 3.6284 \\
\hline
\end{tabular}
Latitude

\begin{tabular}{|c|c|c|}
\hline $\begin{array}{l}\text { World- } \\
\text { gravity } \\
\text { pair }\end{array}$ & $\begin{array}{c}\text { Max } \\
\text { distance } \\
\text { error (nmi) }\end{array}$ & $\begin{array}{c}\text { Final } \\
\text { distance } \\
\text { error (nmi) }\end{array}$ \\
\hline E/PM & 26.4169 & 25.4523 \\
\hline $\mathrm{E} / \mathrm{C}$ & 26.2486 & 25.3090 \\
\hline S/SE & 5.6805 & 5.6805 \\
\hline S/PM & 27.8678 & 22.2815 \\
\hline $\mathrm{S} / \mathrm{C}$ & 27.7636 & 22.1719 \\
\hline$\overline{\text { ENR/FA }}$ & 19.4297 & 17.7707 \\
\hline ENR/C & 19.4297 & 17.7707 \\
\hline SNR/FA & 19.4515 & 18.7161 \\
\hline SNR/C & 19.4515 & 18.7161 \\
\hline
\end{tabular}

Table 7 East from $80^{\circ}$ Latitude

\begin{tabular}{|l|r|r|}
\hline $\begin{array}{l}\text { World- } \\
\text { gravity } \\
\text { pair }\end{array}$ & $\begin{array}{c}\text { Max } \\
\text { distance } \\
\text { error (nmi) }\end{array}$ & $\begin{array}{c}\text { Final } \\
\text { distance } \\
\text { error (nmi) }\end{array}$ \\
\hline E/PM & 47.5590 & 47.5590 \\
\hline E/C & 47.3593 & 47.3593 \\
\hline S/SE & 6.9830 & 0.8452 \\
\hline S/PM & 52.9836 & 52.9836 \\
\hline S/C & 52.7810 & 52.7810 \\
\hline ENR/FA & 22.3458 & 21.6113 \\
\hline ENR/C & 22.3425 & 21.6069 \\
\hline SNR/FA & 22.6633 & 20.8088 \\
\hline SNR/C & 22.6633 & 20.8088 \\
\hline
\end{tabular}

Table $1020^{\circ}$ Track from

\begin{tabular}{|l|r|r|}
\hline $\begin{array}{l}\text { World- } \\
\text { gravity } \\
\text { pair }\end{array}$ & $\begin{array}{c}\text { Equator } \\
\text { Max } \\
\text { distance } \\
\text { error (nmi) }\end{array}$ & $\begin{array}{c}\text { Final } \\
\text { distance } \\
\text { error (nmi) }\end{array}$ \\
\hline E/PM & 45.5110 & 45.5110 \\
\hline E/C & 45.3995 & 45.3995 \\
\hline S/SE & 8.7740 & 8.7740 \\
\hline S/PM & 50.6031 & 50.6031 \\
\hline S/C & 50.4529 & 50.4529 \\
\hline ENR/FA & 22.4026 & 22.4026 \\
\hline ENR/C & 22.4160 & 22.4160 \\
\hline SNR/FA & 30.2520 & 30.2520 \\
\hline SNR/C & 30.2520 & 30.2520 \\
\hline
\end{tabular}

Table 5 West from Equator

\begin{tabular}{|l|r|r|}
\hline $\begin{array}{l}\text { World- } \\
\text { gravity } \\
\text { pair }\end{array}$ & $\begin{array}{c}\text { Max } \\
\text { distance } \\
\text { error (nmi) }\end{array}$ & $\begin{array}{c}\text { Final } \\
\text { distance } \\
\text { error (nmi) }\end{array}$ \\
\hline E/PM & 0.0006 & 0.0006 \\
\hline E/C & 0.0011 & 0.0011 \\
\hline S/SE & 5.3633 & 5.3633 \\
\hline S/PM & 5.3635 & 5.3635 \\
\hline S/C & 5.3644 & 5.3644 \\
\hline ENR/FA & 0.0001 & 0.0001 \\
\hline ENR/C & 0.0007 & 0.0007 \\
\hline SNR/FA & 5.3632 & 5.3632 \\
\hline SNR/C & 5.3632 & 5.3632 \\
\hline
\end{tabular}

Table 8 North-East from Equator

\begin{tabular}{|l|r|r|}
\hline $\begin{array}{l}\text { World- } \\
\text { gravity } \\
\text { pair }\end{array}$ & $\begin{array}{c}\text { Max } \\
\text { distance } \\
\text { error (nmi) }\end{array}$ & $\begin{array}{c}\text { Final } \\
\text { distance } \\
\text { error (nmi) }\end{array}$ \\
\hline E/PM & 27.2739 & 27.2739 \\
\hline E/C & 27.2751 & 27.2751 \\
\hline S/SE & 12.1169 & 12.1169 \\
\hline S/PM & 25.9225 & 25.9225 \\
\hline S/C & 25.8361 & 25.8361 \\
\hline ENR/FA & 17.0438 & 17.0438 \\
\hline ENR/C & 17.0651 & 17.0651 \\
\hline SNR/FA & 29.1041 & 29.1041 \\
\hline SNR/C & 29.1041 & 29.1041 \\
\hline
\end{tabular}

Table $11340^{\circ}$ Track from

\begin{tabular}{|l|r|r|}
\hline $\begin{array}{l}\text { World- } \\
\text { gravity } \\
\text { pair }\end{array}$ & $\begin{array}{c}\text { Equator } \\
\text { Max } \\
\text { distance } \\
\text { error (nmi) }\end{array}$ & $\begin{array}{c}\text { Final } \\
\text { distance } \\
\text { error (nmi) }\end{array}$ \\
\hline E/PM & 45.8332 & 45.8332 \\
\hline E/C & 45.6973 & 45.6973 \\
\hline S/SE & 8.7742 & 8.7742 \\
\hline S/PM & 50.9759 & 50.9759 \\
\hline S/C & 50.8242 & 50.8242 \\
\hline ENR/FA & 21.5533 & 21.5533 \\
\hline ENR/C & 21.5399 & 21.5399 \\
\hline SNR/FA & 14.6577 & 14.6577 \\
\hline SNR/C & 14.6577 & 14.6577 \\
\hline
\end{tabular}


The position errors in the trajectories are explained by analyzing the three major contributors of position error: residual tangential gravity (present in rotating world gravity pairs), Coriolis acceleration (not present in non-rotating world-gravity pairs), or reduced surface fidelity (present in sphere world-gravity pairs). ${ }^{4}$ Five of the world-gravity pairs isolate the impact of each contributor. The E/PM and E/C world-gravity pairs isolate the effects of residual tangent gravity. The ENR/FA and ENR/C world-gravity pairs isolate the effects of missing Coriolis acceleration. The S/SE pair isolates the effects of reduced surface modeling. The remaining subsections examine the impact of those contributors on each route. The discussion focuses on the maximum position errors of the trajectories but the position error at completion is acknowledged when it differs significantly and furthers understanding of the trajectory trends.

\section{A. South from $80^{\circ}$ Latitude}

The South from $80^{\circ}$ Latitude route is the north route of Reference 4 in reverse. Figure 3 and Figure 4 show that the S/SE trajectory maintains close track with E/SE trajectory in both latitude and longitude; therefore, differences in surface modeling between the sphere and ellipsoid have small effect on position error. This is reflected in the position errors of world-gravity pairs that differ only by world shape (e.g. ENR/FA and SNR/FA). In Table 4, the position error differs by no more than $1.5 \mathrm{nmi}$ between world-gravity pairs that differ only by world shape. The ENR/FA trajectory and the trajectory of the other non-rotating world-gravity pairs maintain a close latitude track with the E/SE trajectory but lie to the east by $\sim 0.3^{\circ}$ at completion. This is consistent with the missing Coriolis acceleration that, for the south velocity, is observed as a westerly acceleration in the E/SE trajectory. The E/C and E/PM trajectories drift north of the E/SE trajectory by up to $0.46^{\circ}$ as a result of their residual tangent gravity to the pole. Opposite in direction, the tangent gravity of the S/PM and S/C world gravity pairs drive their trajectories up to $0.44^{\circ}$ south of the E/SE trajectory. The position errors in Table 4 show that the position errors for E/PM, E/C, S/PM, and $\mathrm{S} / \mathrm{C}$ are greater than the errors for ENR/FA, ENR/C, SNR/FA, and SNR/C. Therefore, the residual tangent gravity has a greater affect on position error than the missing Coriolis acceleration. S/SE has the lowest position error. Thus, position errors caused by reduced surface fidelity are less than those caused by wither tangent gravity or Coriolis acceleration. This was the same finding for the north route of Reference 4 (see Table 3).

\section{B. West from Equator}

At the equator, none of the world-gravity pairs experience a residual tangent gravity. Moreover, when traveling due east or west on the equator, the Coriolis acceleration is directed only along the Down axis of a North-East-Down coordinate system. That is, Coriolis acceleration acts along the same axis as gravity but is a tiny fraction of the gravitational force. Since the aircraft is trimmed to a world-relative acceleration of zero, differences in Coriolis acceleration, like differences in gravity, are offset by differences in initial pitch angle that give rise to a counteracting aerodynamic lift. Therefore, the only contributor left to influence aircraft trajectories is reduced surface fidelity of the sphere world model. At the equator the curvature of both sphere and ellipsoid is a circle, so curvature plays no difference. However, the radii of the equators differ. The ellipsoid model uses the mean equatorial radius of the Earth. The radius of the sphere model creates an equal surface area to the ellipsoid; this radius is smaller than the mean equatorial radius (but larger than the polar radius). The effect of reduced surface fidelity is seen in Table 5 and Figure 5 through Figure 8. The position errors for the ellipsoid world-gravity pairs are nearly zero. By contrast, the sphere world-gravity pairs have an error of $5.363 \mathrm{nmi}$. Using simple geometry, the nearly $4800 \mathrm{nmi}$ travel of the aircraft will traverse $\sim 79.82^{\circ}$ of longitude on the ellipsoid (radius $=6378137 \mathrm{~m}$ ) and $\sim 79.91^{\circ}$ of longitude on the sphere (radius $=6371007 \mathrm{~m}$ ). The difference of $0.89^{\circ}$ of longitude equals a distance of $5.369 \mathrm{nmi}$ along the equator of the ellipsoid.

\section{West from $35^{\circ}$ Latitude}

This route begins at the latitude where the geocentric radius of the ellipsoid surface equals the radius of the sphere surface. The initial velocity of the aircraft is due west. Thus, the route starts with a Coriolis acceleration directed north. However, the aircraft does not remain on its west track. It drifts south along a great circle route. As it does, the Coriolis acceleration will have components directed both west and north, but the Coriolis acceleration will also decrease in magnitude as aircraft approaches the equator. As depicted in Figure 11, the reference E/SE trajectory does track north of the ENR/FA trajectory and the trajectories of the other non-rotating world-gravity pairs as predicted by the Coriolis acceleration. Furthermore, Figure 12 shows that the E/SE trajectory does track west of trajectories for non-rotating world-gravity pairs. The ENR/FA and ENR/C trajectories drift up to $0.189^{\circ}$ south of the E/SE trajectory and up to $0.129^{\circ}$ east. The SNR/FA and SNR/C trajectories drift up to $0.248^{\circ}$ south and $0.099^{\circ}$ east. The larger south drift and smaller east drift of SNR/FA and SNR/C can be attributed to the addition of reduced surface fidelity effects as revealed in the S/SE trajectory. The S/SE trajectory lies close to the E/SE trajec- 
tory but lies slightly the south by up to $0.065^{\circ}$ and slightly west by up to $0.081^{\circ}\left(0.031^{\circ}\right.$ at completion $)$. The E/PM and $\mathrm{E} / \mathrm{C}$ trajectories show that residual tangent gravity induces both a north drift of up to $0.3^{\circ}$ and an east drift of up to $0.1^{\circ}$. With tangent gravity directed to the equator, the S/PM and S/C trajectories drift south by up to $0.4^{\circ}$ and drift west by up to $0.165^{\circ}$. The S/PM and S/SE trajectories have a slightly larger drift than explained by tangent gravity because reduced surface fidelity also drives these trajectories to the south and west. Table 6 shows the resulting position errors. Again, position errors caused by tangent gravity in rotating world-gravity pairs (except S/SE) are greater than the errors related to missing Coriolis acceleration in non-rotating world-gravity pairs. Position errors caused by reduced surface fidelity (S/SE) are less than errors caused by tangent gravity or Coriolis acceleration.

\section{East from $80^{\circ}$ Latitude}

This route examines trajectories that start at an altitude where the geocentric radius to the surface is smaller on the ellipsoid than the sphere model. The initial velocity of the aircraft is due east. Thus, the initial Coriolis acceleration is directed south. As with the West from $35^{\circ}$ Latitude route, the aircraft turns south to follow a great circle route. Thus, the Coriolis acceleration will have components directed south and west through most of the trajectory. Figure 15 and Figure 16 show the latitude and longitude of the trajectories, respectively. The S/SE trajectory follows the E/SE trajectory closely, showing little error due to reduced surface fidelity; nevertheless, the trajectory does drift south up to $0.11^{\circ}$ and east up to $0.127^{\circ}$ before ending with drifts of less than $0.008^{\circ}$ south and $0.012^{\circ}$ east. The trajectory of ENR/FA and the other non-rotating world-gravity pairs lie east of E/SE consistent with the observed Coriolis acceleration. The trajectories of non-rotating world-gravity pairs also lies to the north of E/SE as expected from the Coriolis acceleration; however, this north drift is dwarfed by the latitude differences among the rotating world-gravity pairs. The E/PM and E/C trajectories show that tangent gravity contributes to a north drift of up to $0.79^{\circ}$ and a west drift of up to $0.07^{\circ}$. With tangent gravity in the opposite direction, the S/PM and S/C trajectories drift south up to $0.88^{\circ}$ and drift east up to $0.146^{\circ}\left(0.07^{\circ}\right.$ by the end of the trajectory). As with the West from $35^{\circ}$ Latitude route, the slightly larger drift south in the S/PM and S/C trajectories is a combination of drift due to tangent acceleration and drift due to reduced surface fidelity. Table 7 shows the resulting errors. Again there is no change in the error trends. Position errors due to tangent gravity in rotating world-gravity pairs (except S/SE) are greater than the errors related to missing Coriolis acceleration in non-rotating world-gravity pairs. Position errors caused by reduced surface fidelity (S/SE) are less than errors caused by tangent gravity or Coriolis acceleration.

\section{E. North-East from Equator and North-West from Equator}

The North-East from Equator and North-West from Equator routes are discussed together because they have mirrored initial conditions. The North-East from Equator route takes a $45^{\circ}$ track and the North-West from Equator route takes a $-45^{\circ}$ track. With an initial east (or west) velocity and an initial north velocity, North and East components of Coriolis acceleration emerge as latitude increases. (For both routes, the East component of Coriolis acceleration points east.) However, as the aircraft follows a great circle route, the velocity turns more easterly (or westerly) and the Coriolis acceleration will turn to the south (or north) toward the end of the trajectory. Figure 19 and Figure 20 show the latitude and longitude trends in the trajectories of the North-East from Equator route. In this route, the S/SE trajectory visibly departs from the E/SE trajectory. Reduced surface fidelity causes the S/SE trajectory to drift north up to $0.2^{\circ}$ and drift west up to $0.095^{\circ}$. The residual tangent gravity causes the E/PM and E/C trajectories to drift north up to $0.26^{\circ}$ and drift east up to $0.52^{\circ}$. This is the first route where the tangent gravity induces a greater drift in longitude than in latitude. Residual tangent gravity causes drift in the opposite direction for the S/PM and $\mathrm{S} / \mathrm{C}$ trajectories. However, since S/PM and S/C trajectories are also subject to drift caused by reduced surface fidelity, the combined effects indicate that the south drift will shrink and the west drift should increase slightly. In fact, the S/PM and S/C trajectories drift south by up to $0.216^{\circ}$ which shrinks to $0.07^{\circ}$ at completion. These trajectories also drift west by up to $-0.58^{\circ}$, comparable to the drift of the $\mathrm{E} / \mathrm{PM}$ and $\mathrm{E} / \mathrm{C}$ trajectories. As predicted by the direction of the Coriolis acceleration, the ENR/FA and ENR/C trajectories drift north and west of the E/SE trajectory by up to $0.28^{\circ}$ and $0.11^{\circ}$ respectively. Because the SNR/FA and SNR/C trajectories experience drift due to a combination of reduced surface fidelity and missing Coriolis acceleration, these trajectories have a larger north and west drift of up to $0.48^{\circ}$ and $0.20^{\circ}$ respectively. Table 8 shows the resulting position errors. The error trends among the ellipsoid world-gravity pairs remain the same; ENR/FA and ENR/C produce lower errors than E/PM and E/C. However, the interplay among the contributors to position error produces a new trend for sphere world-gravity pairs. S/SE continues to produce the lowest position error; however, S/PM and S/C produce lower errors than SNR/FA and SNR/C. Furthermore, S/PM and S/C produce slightly lower errors than E/PM and E/C.

Compared to the North-East from equator route, Figure 23 and Figure 24 show some mirrored trends in the North-West from Equator route, but the interplay among error contributors produces different results for the sphere world-gravity pairs. The $\mathrm{S} / \mathrm{SE}$ trajectory again drifts north by up to $0.20^{\circ}$ but, mirroring the North-East from Equa- 
tor route, drifts $0.98^{\circ}$ east. These are the approximate position errors due to reduced surface fidelity. The Coriolis acceleration is perpendicular to the velocity vector. Therefore, the aircraft velocity that is mirrored from east to west produces a Coriolis acceleration that is mirrored from south to north. The East component of Coriolis acceleration remains pointing east. As predicted by the direction of the Coriolis acceleration, the ENR/FA and ENR/C trajectories drift south of the $\mathrm{E} / \mathrm{SE}$ trajectory by up to $0.257^{\circ}$ and drift west by up to $0.09^{\circ}$. The tangent gravity causes the $\mathrm{E} / \mathrm{PM}$ and $\mathrm{E} / \mathrm{C}$ trajectories to drift north by up to $0.269^{\circ}$ and, in mirror to the North-East from Equator route, drift west by up to $0.53^{\circ}$. Acting in the opposite direction, the tangent gravity induces a south drift and east drift in the $\mathrm{S} / \mathrm{PM}$ and S/C trajectories. Again, the tangent gravity and surface modeling error combine to produce a reduced southern drift of up to $0.22^{\circ}\left(0.08^{\circ}\right.$ at completion $)$ in the S/PM and S/C trajectories and a slight increase in east drift of up to $0.60^{\circ}$. The drift in the SNR/FA and SNR/C trajectories is a combination of missing Coriolis acceleration and reduced surface fidelity. Unlike the North-East from Equator route, the south drift due to missing Coriolis acceleration in the North-West from Equator route subtracts from the north drift due to reduced surface fidelity. Moreover, the east drift due to reduced surface fidelity nearly eliminates the west drift due to missing Coriolis acceleration. The result is that the SNR/FA and SNR/C trajectories drift south by a reduced maximum of $0.107^{\circ}\left(0.06^{\circ}\right.$ at completion) and drift west by a small $0.013^{\circ}$. Table 9 shows the resulting position errors. Again, the trend among ellipsoid world-gravity pairs remains unchanged. However, the interplay between reduced surface fidelity, tangent gravity, and Coriolis accelerations, creates a new trend for the sphere world-gravity pairs. First, the smallest error among all the sphere world-gravity pairs is no longer S/SE but SNR/FA and SNR/C. S/PM and S/C still produce a larger error that $\mathrm{S} / \mathrm{SE}$ but the difference is less than $3 \mathrm{nmi}$. Lastly, all of the sphere world-gravity pairs produce less position error than the ellipsoid world-gravity pairs.

\section{F. $20^{\circ}$ Track from Equator and $340^{\circ}$ Track from Equator}

The $20^{\circ}$ Track from Equator and $340^{\circ}$ Track from Equator routes are another pair of routes with mirrored initial conditions. The North-East from Equator and North-West from Equator routes in the previous section produce trajectories that spend more time in the region where the surface of the sphere lies at a smaller geocentric radius than the surface of the ellipsoid. The $20^{\circ}$ Track from Equator and $340^{\circ}$ Track from Equator attempt to produce trajectories that spend nearly equal time in regions where the geocentric radius of the sphere is smaller and larger than the geocentric radius of the ellipsoid surface. The resulting trajectory of the E/SE pair spends $\sim 5 \frac{1}{2}$ hours in the region where the geocentric radius of the ellipsoid surface is larger and the remaining $\sim 61 / 2$ hours in the region where the geocentric radius of the ellipsoid surface is smaller. Like the previous two routes, the Coriolis acceleration will have both a North and East component in these routes. Coriolis acceleration is directed east and south for $20^{\circ}$ Track from Equator and directed east and north for $340^{\circ}$ Track from Equator. The East component will predominate early in the route, but, as the trajectory turns east (or west) toward the end of the route, the North component will become dominant. Figure 27 and Figure 28 show the latitude and longitude of the trajectories in the last hour of travel. The S/SE trajectory remains close in latitude to the E/SE reference trajectory but has a noticeable west drift in longitude. The maximum latitude drift of the S/SE directory is $0.13^{\circ}$ south at mid-route but the trajectory recovers to drift only $0.08^{\circ}$ north at completion. The maximum longitude drift is $0.33^{\circ}$ west and is near this value at completion. Thus, reduced surface fidelity induces a west drift in trajectories on the sphere world. Residual tangent gravity causes the E/PM and E/C trajectories to drift north by up to $0.53^{\circ}$ and drift east by up to $1.68^{\circ}$. Thus, the affect of residual tangent gravity on S/PM and S/C trajectories is to drive the trajectories to the south and west. The west drift for theses trajectories is driven further west by the effect of reduced surface fidelity. Maximum drift for the $\mathrm{S} / \mathrm{PM}$ and $\mathrm{S} / \mathrm{C}$ trajectories is $0.596^{\circ}$ south and $1.98^{\circ}$ west. The ENR/FA and ENR/C trajectories drift to the north by up to $0.335^{\circ}$ and west by up to $0.474^{\circ}$ as predicted by Coriolis acceleration. In the SNR/FA and SNR/C trajectories, the effects of Coriolis acceleration and reduced surface fidelity combine to bump up the drift north and increase the drift west. The north drift is up to $0.413^{\circ}$ and the west drift is up to $0.808^{\circ}$. Table 10 shows the resulting position errors. S/SE produces the lowest error. The remaining rotating world-gravity pairs (E/PM, E/C, S/PM, and S/C) produce larger errors than the non-rotating world-gravity pairs. Pairs using a sphere model produce larger errors than the ellipsoid pair using the same gravity/gravitation model.

Figure 31 and Figure 32 show the latitude and longitude of trajectories in the last hour of travel on the $340^{\circ}$ Track from Equator route. The S/SE trajectory again drifts up to $0.13^{\circ}$ south in mid-route but recovers to drift $0.07^{\circ}$ north at completion. The S/SE trajectory also drifts up to $0.34^{\circ}$ east, mirroring the west drift under the $20^{\circ}$ Track from equator route. Thus, reduced surface fidelity causes minimal drift south but induces an east drift. The E/PM and $\mathrm{E} / \mathrm{C}$ trajectories show that residual tangent gravity causes a drift north up to $0.547^{\circ}$ and a drift west of up to $1.710^{\circ}$ (mirroring the east drift in $20^{\circ}$ Track from Equator route). The tangent gravity for S/PM and $\mathrm{S} / \mathrm{C}$ is in the opposite direction and drives those trajectories south and east. In the S/PM and S/C trajectories, the combined effects of residual tangent gravity and reduced surface fidelity push the trajectory up to $0.611^{\circ}$ south and up to $2.018^{\circ}$ 
east. As predicted by the Coriolis acceleration, the ENR/FA and ENR/C trajectories drift up to $0.318^{\circ}$ degrees south and $0.475^{\circ}$ west. In the SNR/FA and SNR/C trajectories, the effect of Coriolis acceleration and surface modeling error combine to reduce the west drift throughout the trajectory and nudge the south drift to the north at completion. The SNR/FA and SNR/C directories drift up to $0.240^{\circ}$ south and up to $0.148^{\circ}$ west. Table 11 presents the resulting position errors. The trends are the same as the $20^{\circ}$ Track from Equator route with one exception. The SNR/FA and SNR/C routes produce a smaller error than the ENR/FA and ENR/C routes.

\section{Conclusions}

This study investigates the impact of world-gravity pairs on a free-flying subsonic aircraft in straight and level flight. Reference 4 presented initial results on a north route with this aircraft and found that non-rotating worldgravity pairs produced lower errors than the rotating world-gravity pairs. This study examines eight more routes: a south route starting at $\sim 80^{\circ}$ latitude, a west route along the equator, and a west route at $\sim 35^{\circ}$ latitude, an east route at $\sim 80^{\circ}$ latitude, a north-east route starting at the equator, a north-west route starting at the equator, a $20^{\circ}$ track route starting at the equator, and a $340^{\circ}$ track route starting at the equator. Before analyzing the trajectories on these routes with various world-gravity pairs, the application of the symmetric ellipsoid gravitation model on the sphere was re-examined. Reference 4 took advantage of the equivalence of geocentric and geodetic coordinates on the sphere to feed the geocentric coordinates of the aircraft directly into the symmetric ellipsoid gravitation equations. This approach creates an identical gravitation field in an Earth-fixed, Earth-centered (ECEF) frame for both the sphere and ellipsoid models. At the same geodetic coordinates, however, the gravitation vectors will differ because the ECEF coordinates of those geodetic coordinates differ between ellipsoid and sphere models of the Earth. An alternate approach was proposed that would compute identical gravitation vectors at the same geodetic location on both the sphere and ellipsoid. In this approach, the symmetric ellipsoid gravitation model always treats the geodetic coordinates as if they are referenced to the ellipsoid model of the Earth (even when the coordinates are referenced to a sphere). This creates a gravitation field this is identical between sphere and ellipsoid from the perspective of an observer on the surface. When this surface-fixed gravitation field is applied to the rotating sphere model of the Earth (i.e. the S/SE world-gravity pair), the position errors of the aircraft trajectories are reduced by up to an order of magnitude. The reduced errors occur because the residual tangent gravity produced using the ECEF gravitation field is almost non-existent when using the surface-fixed gravitation field. With the elimination of the residual tangent gravity, the S/SE world-gravity pair isolates position errors due to reduced surface fidelity of the sphere. This completes the set of world-gravity pairs that isolate each of the contributors to position errors relative to the reference $\mathrm{E} / \mathrm{SE}$ world gravity pair. Those contributors are residual tangent gravity (isolated by the E/PM and E/C pairs), Coriolis acceleration (isolated by the ENR/FA and ENR/C pairs), and reduced surface fidelity (isolated by the S/SE pair). This core set of world-gravity pairs was used as a basis for analyzing the trajectories in each of the eight routes.

The north route of Reference 4 established an initial order of increasing position error (decreasing trajectory fidelity) for comparison:

- S/SE world gravity pair

- ENR/FA and ENR/C world gravity pairs

- SNR/FA and SNR/C world gravity pairs

- $\mathrm{E} / \mathrm{PM}$ and $\mathrm{E} / \mathrm{C}$ world gravity pairs

- S/PM and S/C world gravity pairs

This trend recurs in half of the eight routes: South from $80^{\circ}$ Latitude, West from $35^{\circ}$ Latitude, East from $80^{\circ}$ Latitude, and $20^{\circ}$ Track from Equator. However, the position errors in the routes originating from $80^{\circ}$ latitude differ by less than $0.3 \mathrm{nmi}$ among the non-rotating world-gravity pairs. In the West from Equator route, residual tangent acceleration does not exist and Coriolis acceleration (directed only upward) does not affect position errors. Only the world-gravity pairs using a sphere model produce a position error, and that error results from the difference in equatorial radii between the ellipsoid and sphere world models. In the North-East from Equator route, the missing Coriolis acceleration and reduced surface fidelity combine to drive the position errors of the SNR/FA and SNR/C trajectories to the highest among world-gravity pairs. On the other hand, tangent residual gravity and reduced surface fidelity counteract to reduce the position error of the S/PM and S/C trajectories so that they are less than the position errors of the E/PM and E/C trajectories. The S/PM and S/C trajectories see the same effect in the North-East from Equator route. However, in this route, the missing Coriolis acceleration and reduced surface fidelity also counteract to produce position errors for the SNR/FA and SNR/C trajectories that are the lowest among the world-gravity pairs. Finally, the $340^{\circ}$ Track from Equator route produces counteracting effects from missing Coriolis acceleration and 
reduced surface fidelity that reduce the position error of the SNR/FA and SNR/C trajectories below the position error of the ENR/FA and ENR/C trajectories.

The results show that the order of position error among the world-gravity pairs can depend on the route. However, some general trends do emerge for simulations of a subsonic aircraft. In all but one route, the S/SE gravity pair produces the lowest position error. Thus, when reducing fidelity by using a sphere model of the Earth, S/SE is the first choice. When reducing the fidelity of the gravity or gravitation model, ENR/FA is a good general choice. It outperforms or equals the E/PM, S/PM, and SNR/FA pairs in all but one route (two routes if judging based on distance error at completion). SNR/FA should be the second choice; it outperforms or equals the E/PM and S/PM world gravity pairs in all but one route. The pairs using constant gravity or constant gravitation models perform do perform equally to counterparts using point-mass gravitation or free-air reduction gravity. However, this equality of performance may be an artifact of straight and level flight, so world-gravity pairs using point-mass gravitation and free-air reduction are given preference in this recommendation.

There are limitations to this analysis that require further examination. Coriolis acceleration is a function of vehicle velocity, so results presented here cannot be extended to supersonic or hypersonic vehicles without further investigation. Also, Reference 2 recommends against using the free-air reduction model above $15.9 \mathrm{~km}(52,000 \mathrm{ft})$. Thus, the results presented here cannot extend to high-altitude vehicles without further study. 


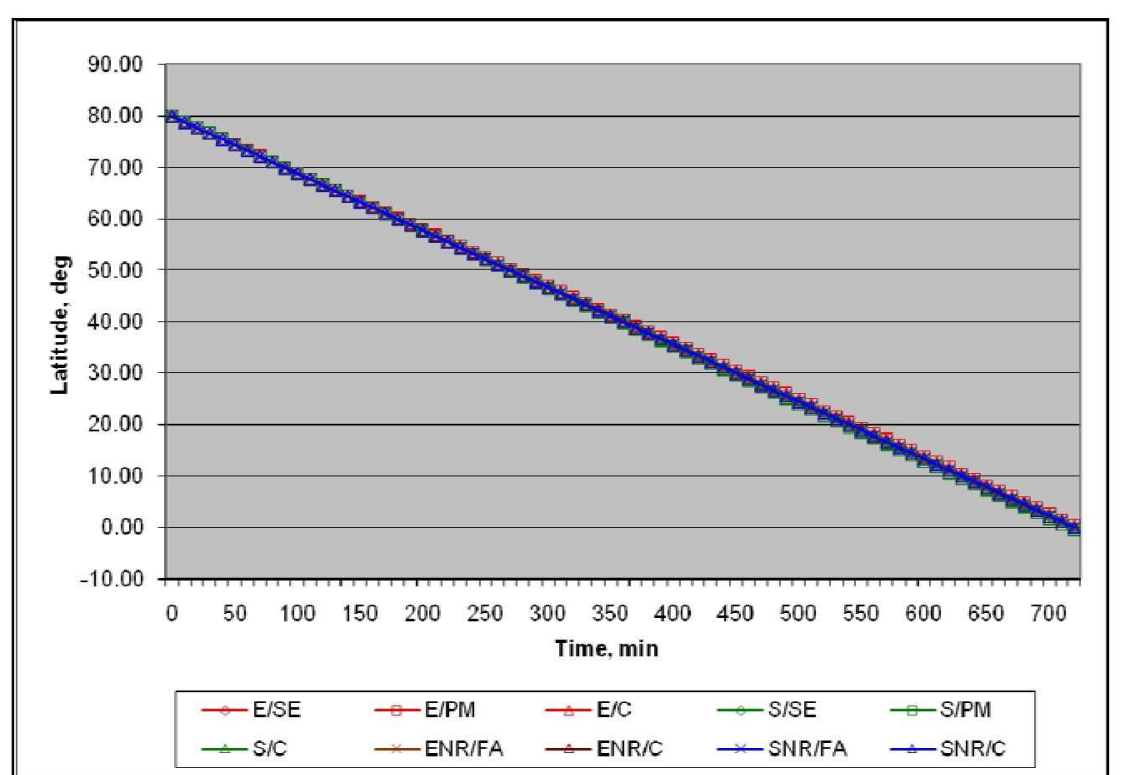

Figure 1 South from $80^{\circ}$ Latitude - Latitude vs. Time

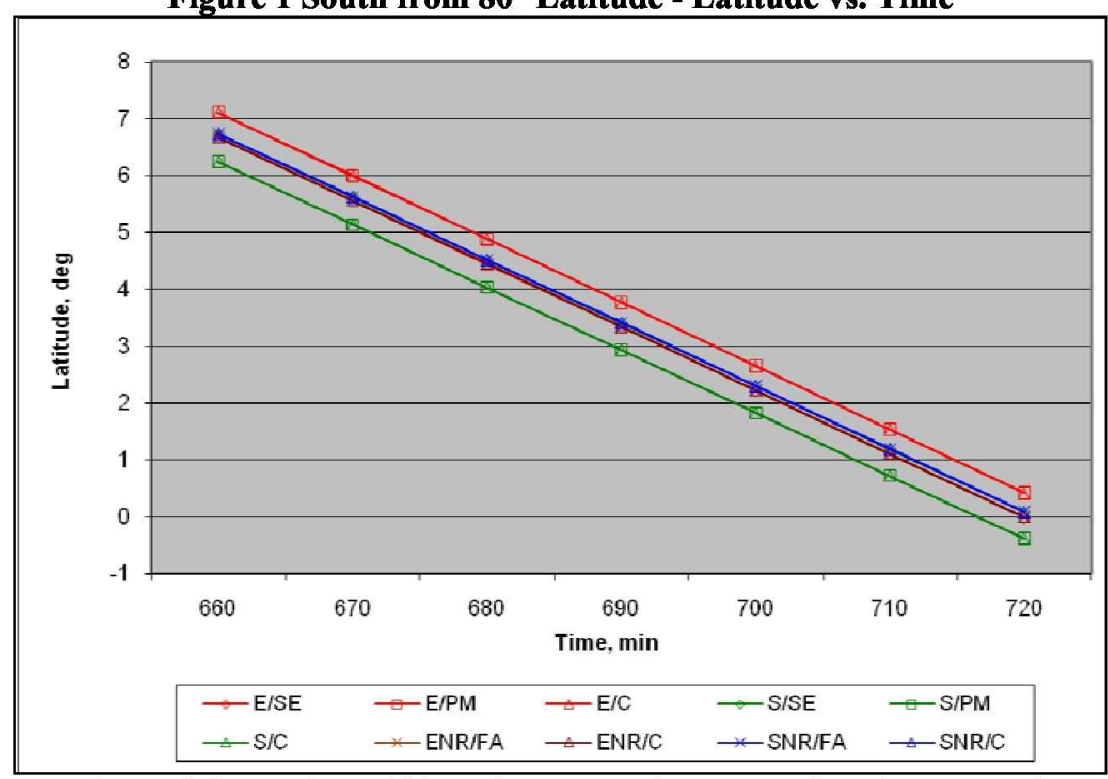

Figure 3 South from $80^{\circ}$ Latitude - Latitude vs. Time (Last Hour)

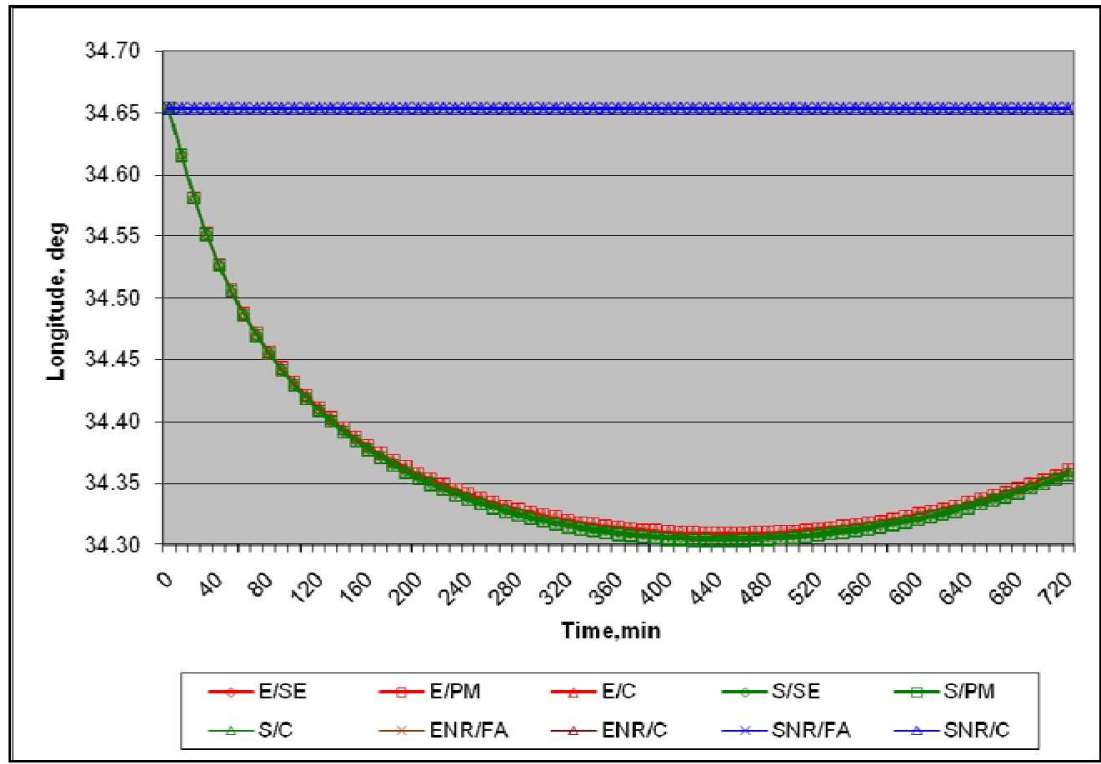

Figure 2 South from $80^{\circ}$ Latitude - Longitude vs. Time

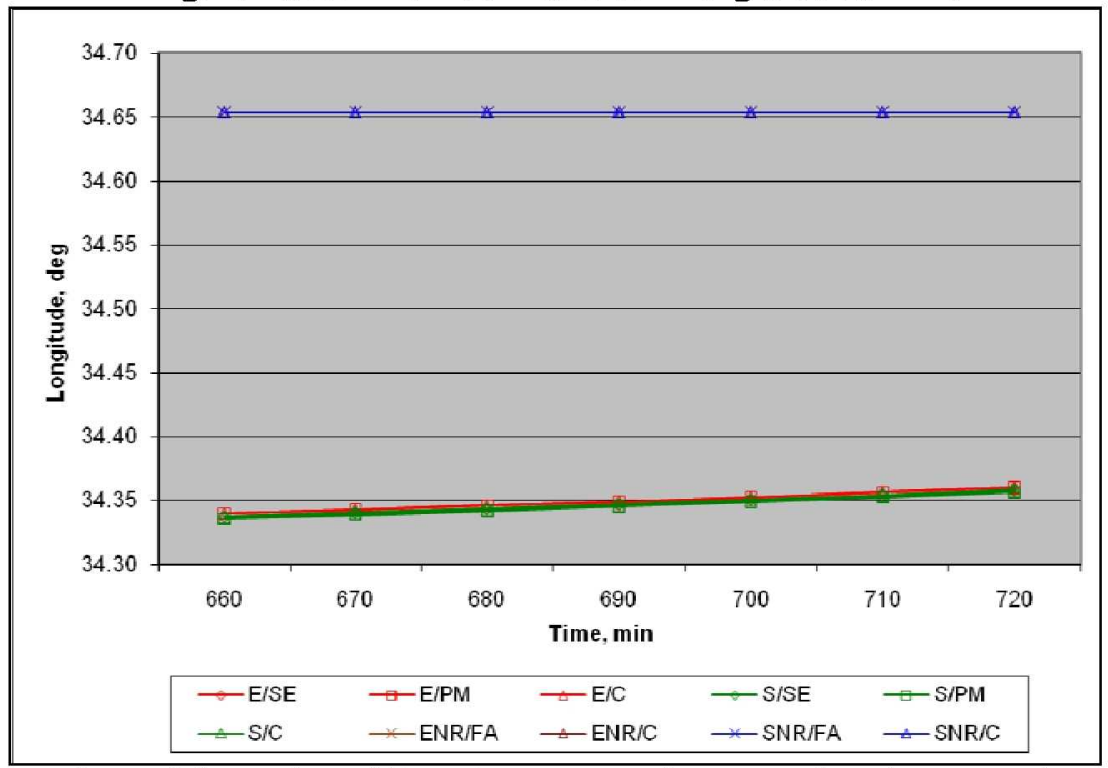

Figure 4 South from $80^{\circ}$ Latitude - Longitude vs. Time (Last Hour) 


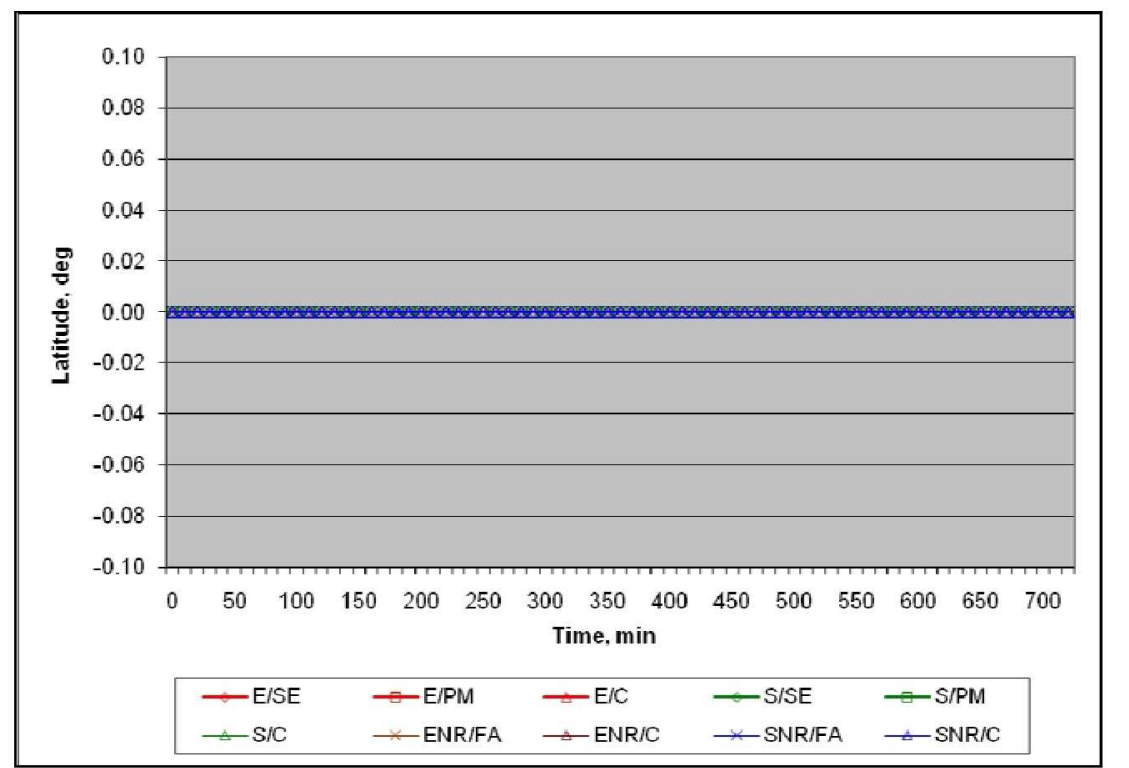

Figure 5 West from Equator - Latitude vs. Time

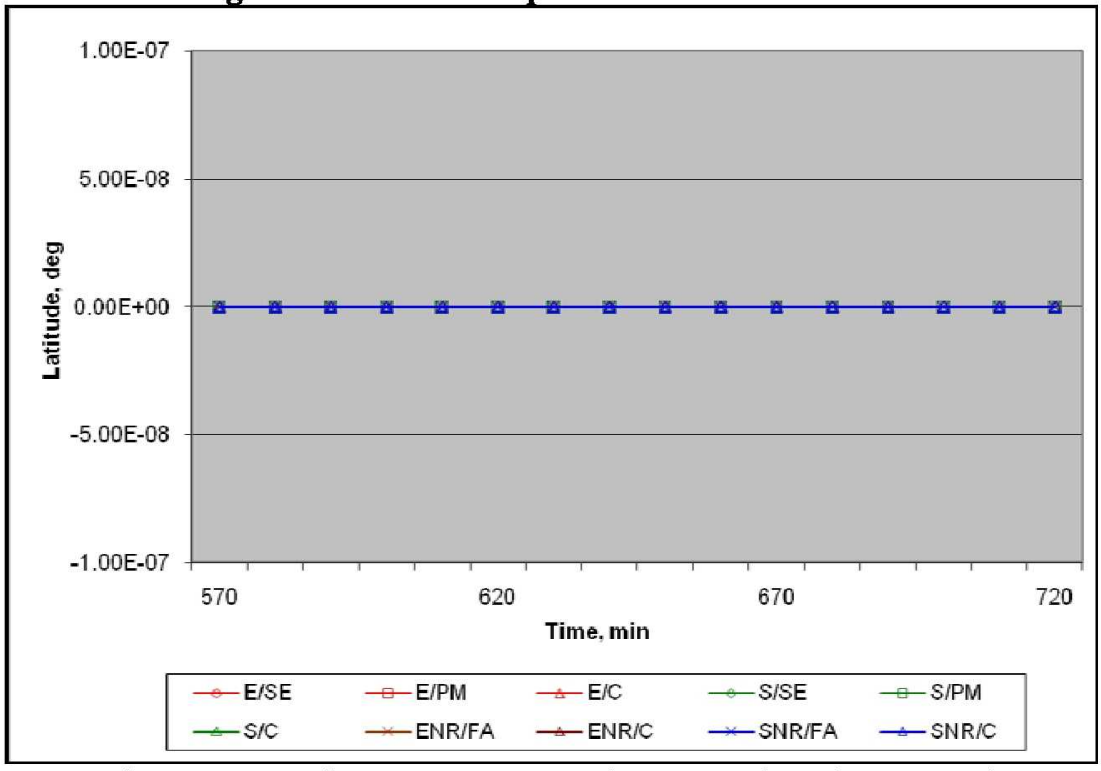

Figure 7 West from Equator - Latitude vs. Time (Last Hour)

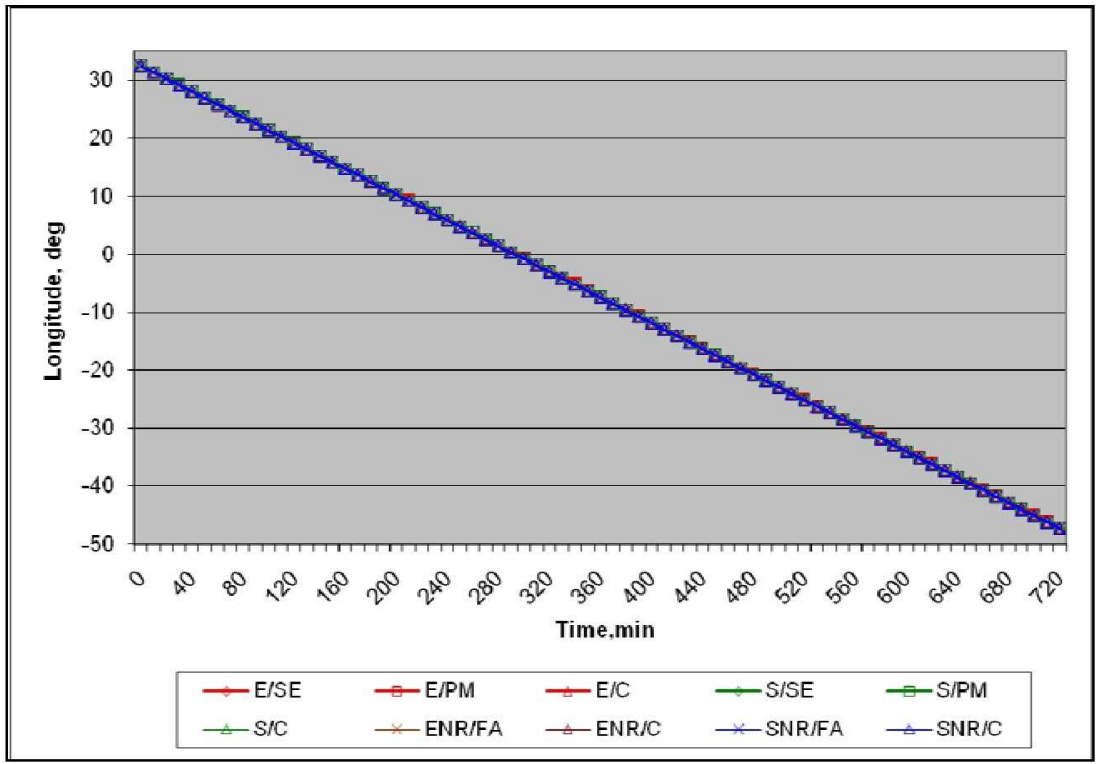

Figure 6 West from Equator - Longitude vs. Time

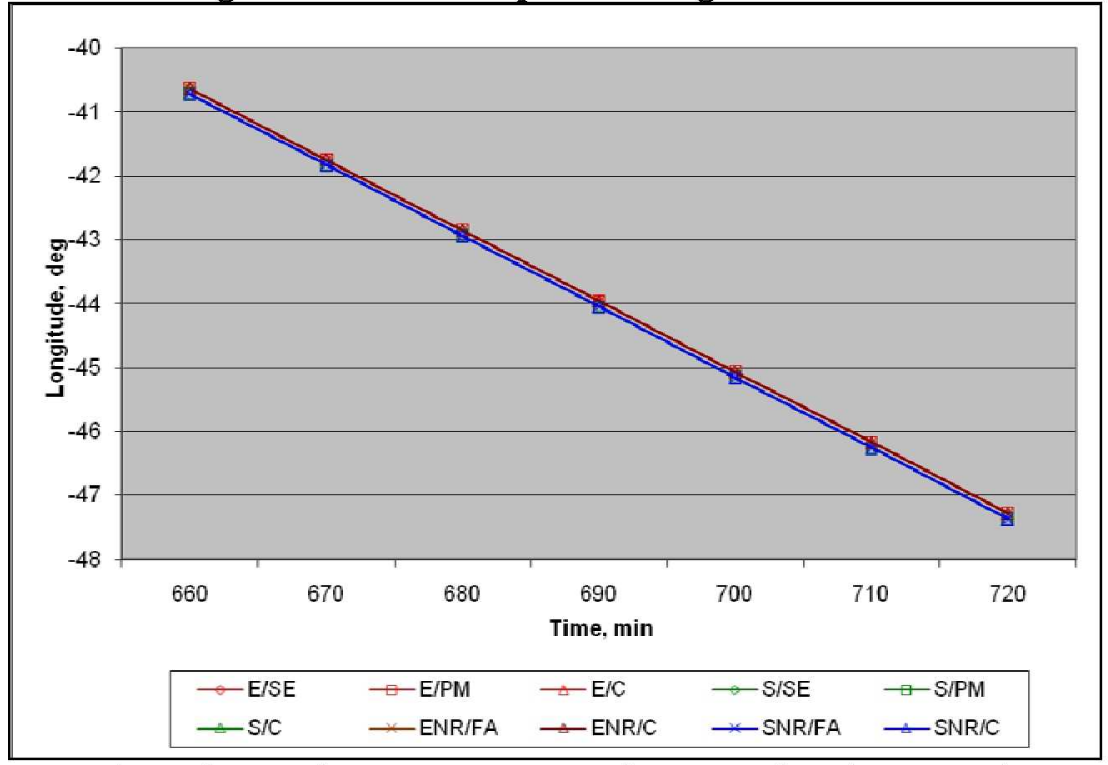

Figure 8 West from Equator - Longitude vs. Time (Last Hour) 


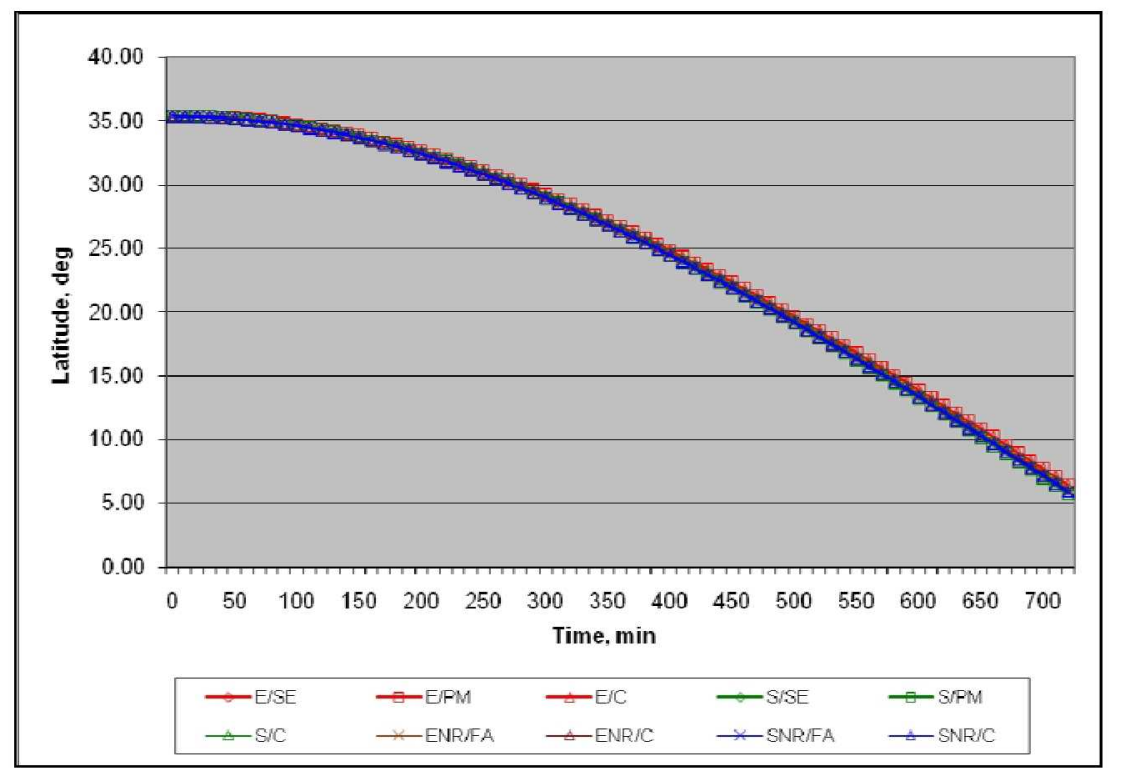

Figure 9 West from $35^{\circ}$ Latitude - Latitude vs. Time

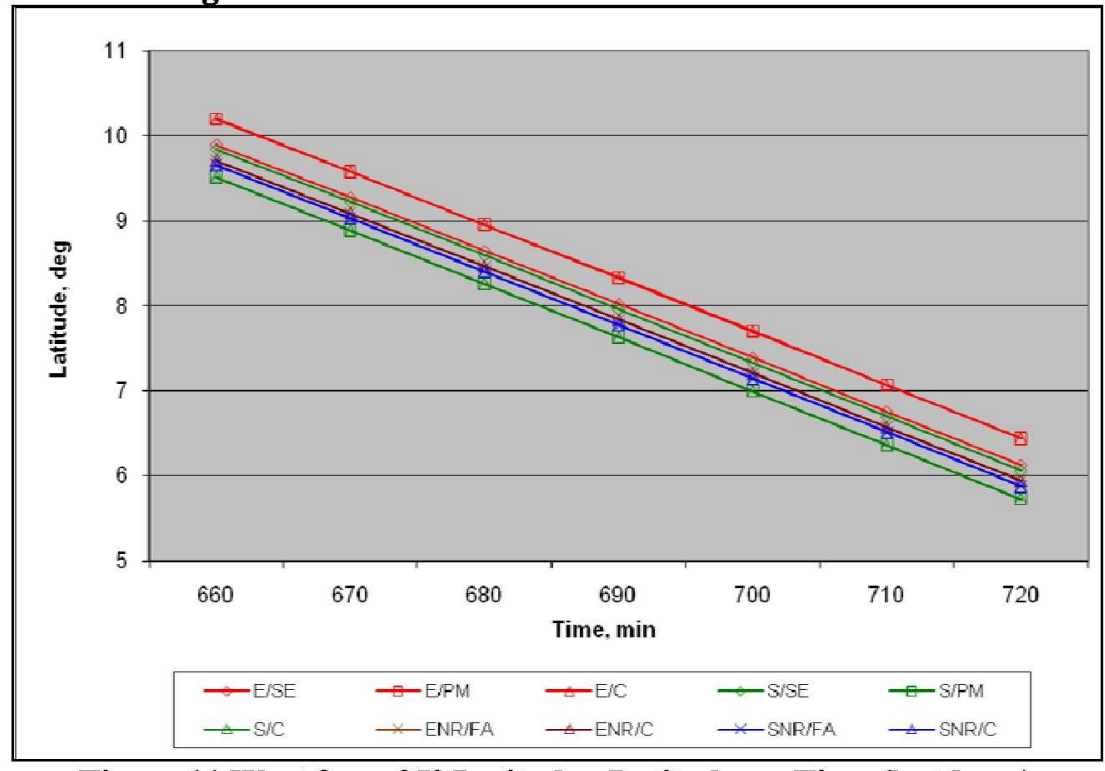

Figure 11 West from $35^{\circ}$ Latitude - Latitude vs. Time (last hour)

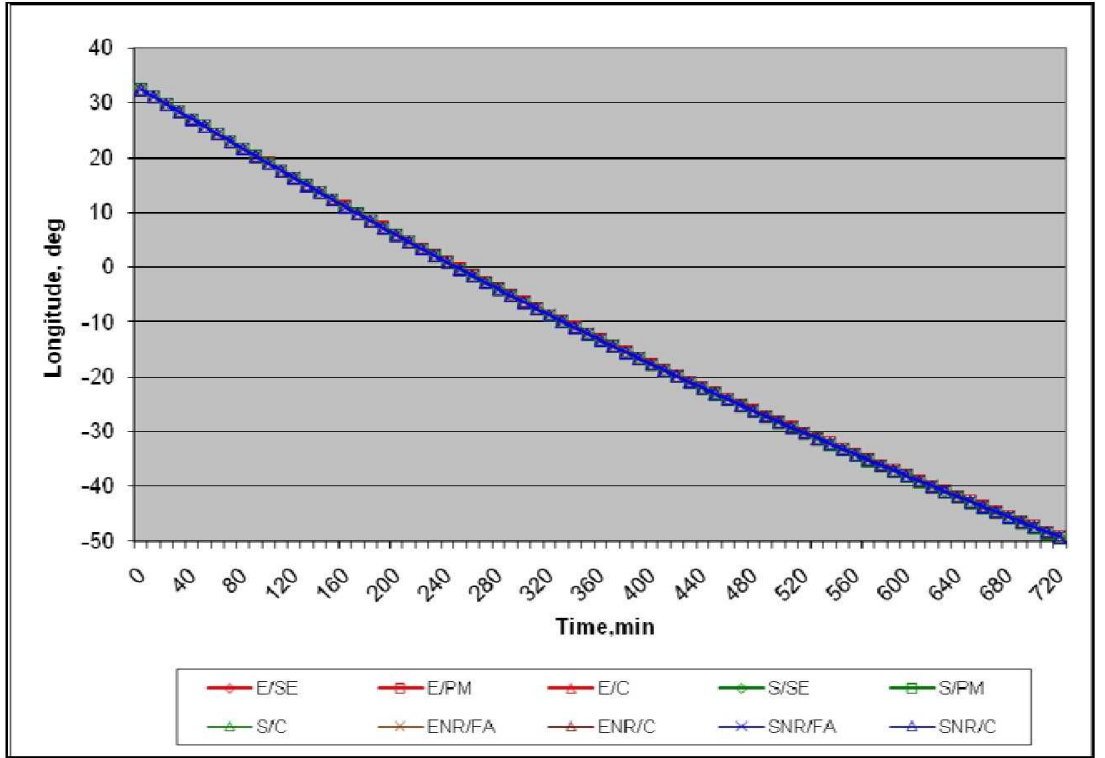

Figure 10 West from $35^{\circ}$ Latitude - Longitude vs. Time

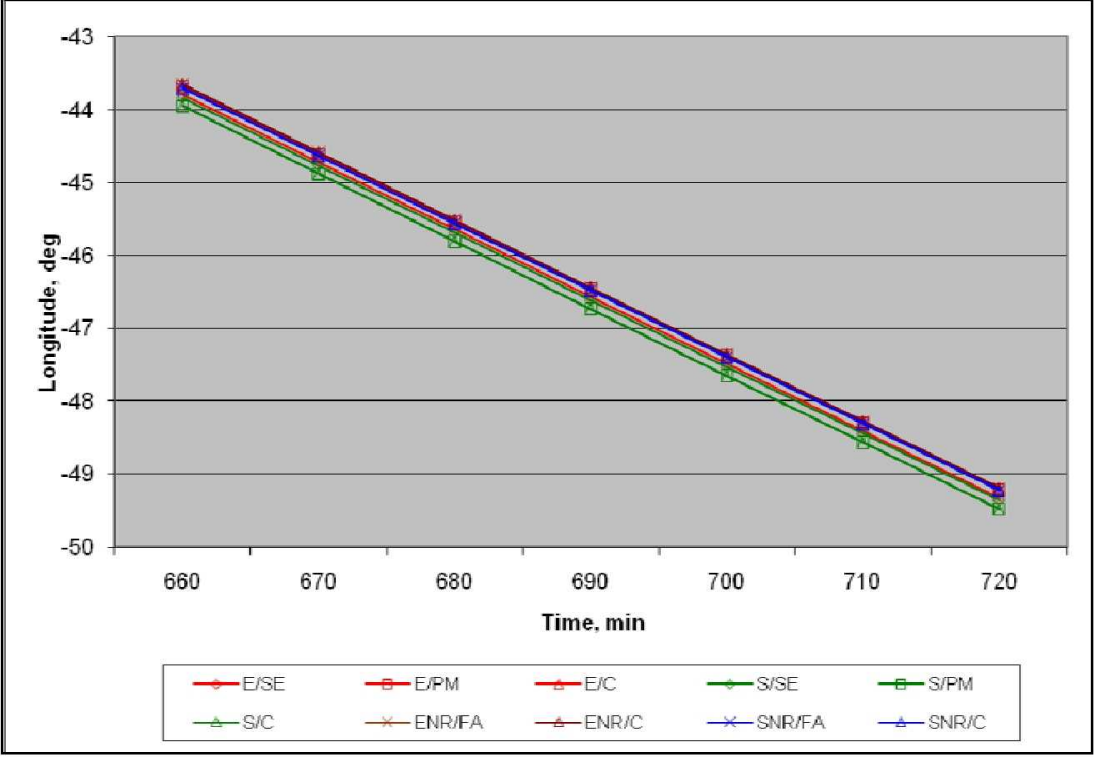

Figure 12 West from $35^{\circ}$ Latitude - Longitude vs. Time (Last Hour) 


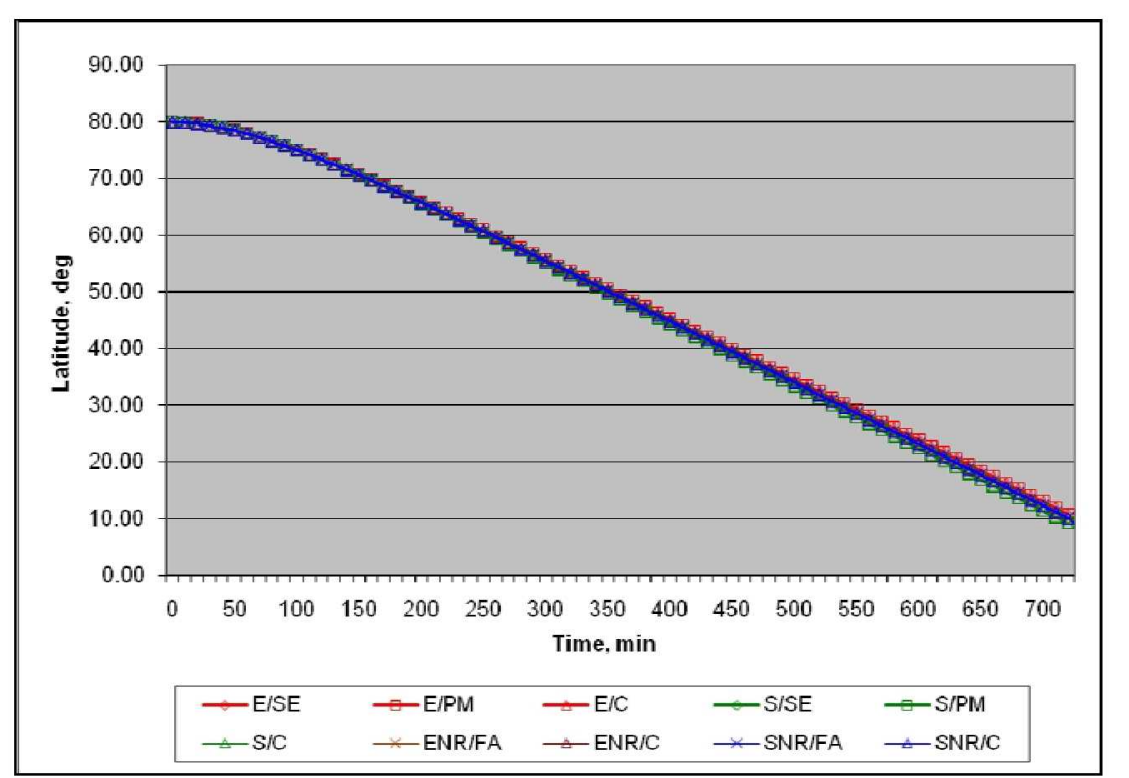

Figure 13 East from $80^{\circ}$ Latitude - Latitude vs. Time

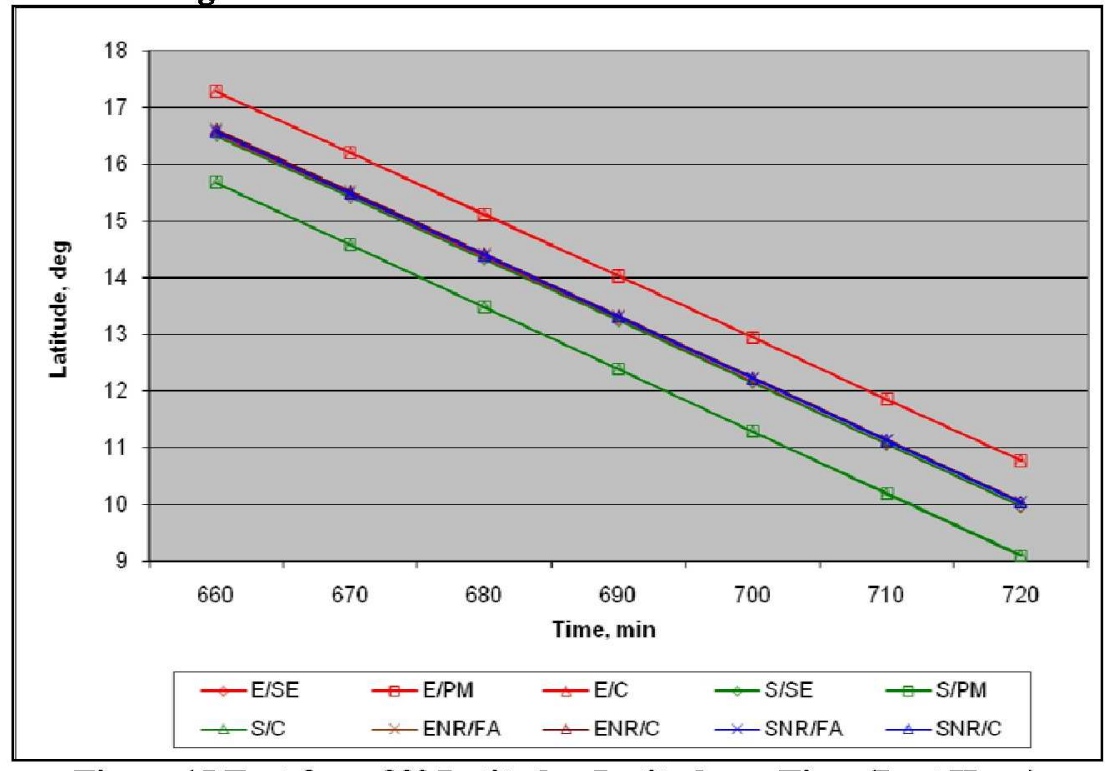

Figure 15 East from $80^{\circ}$ Latitude - Latitude vs. Time (Last Hour)

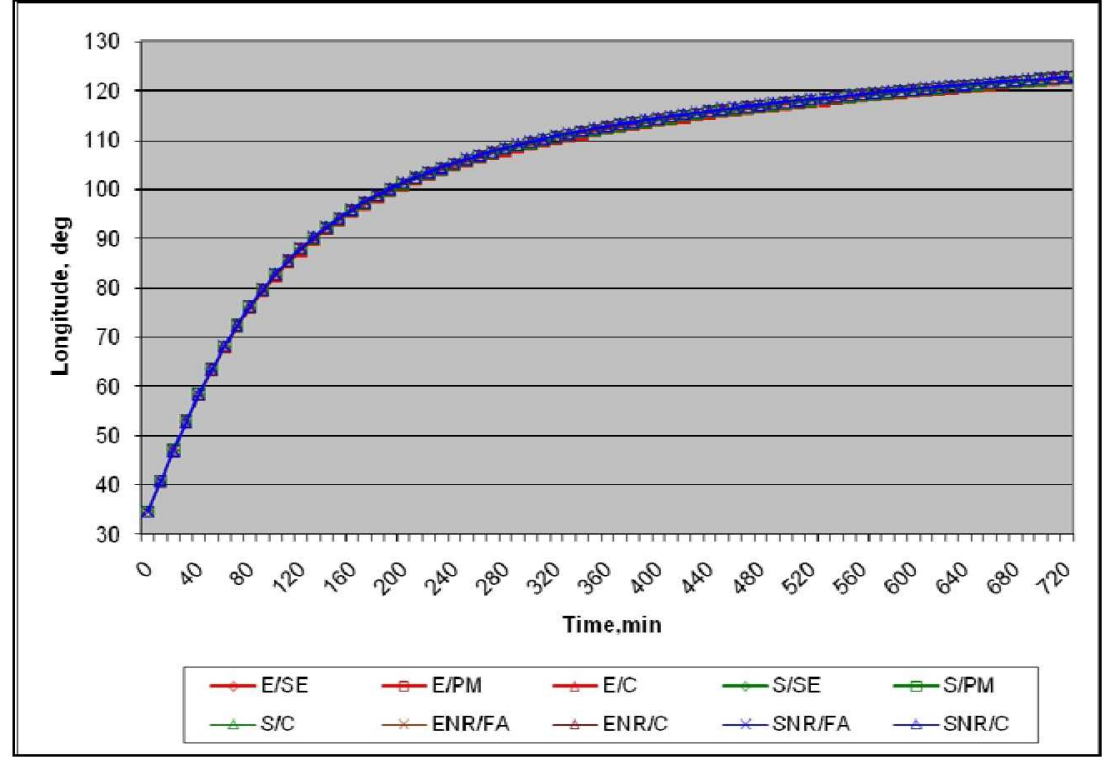

Figure 14 East from $80^{\circ}$ Latitude - Longitude vs. Time

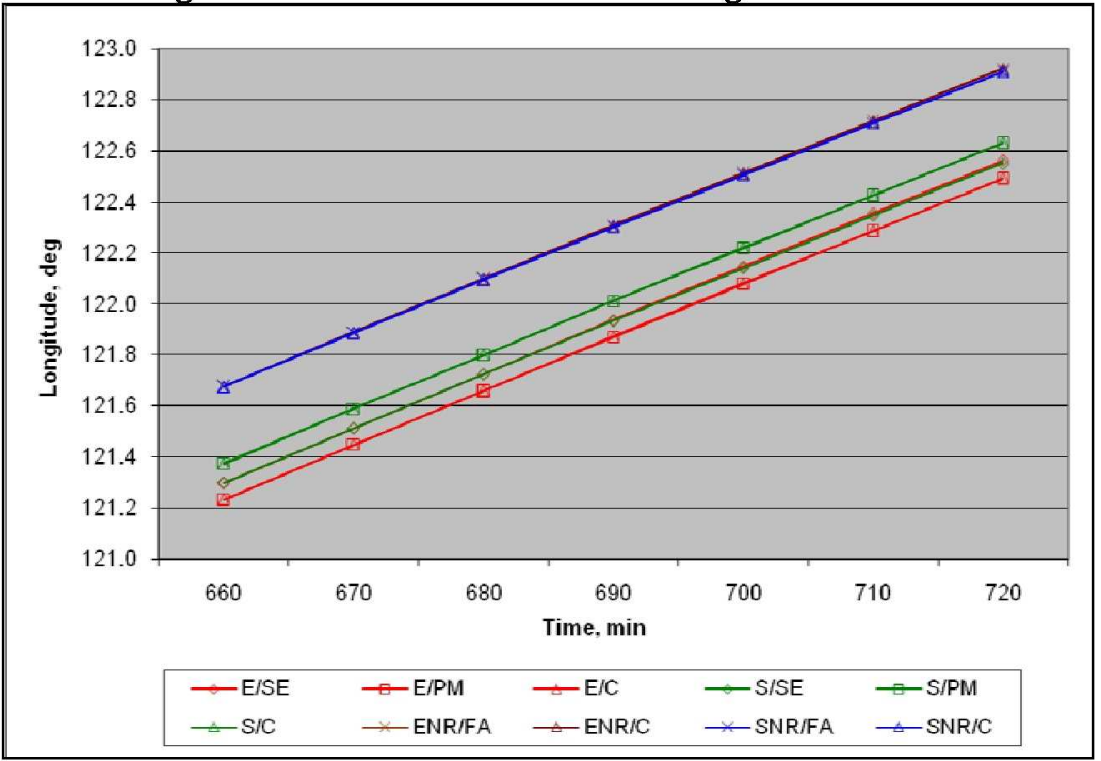

Figure 16 East from $80^{\circ}$ Latitude - Longitude vs. Time (Last Hour) 17

American Institute of Aeronautics and Astronautics 


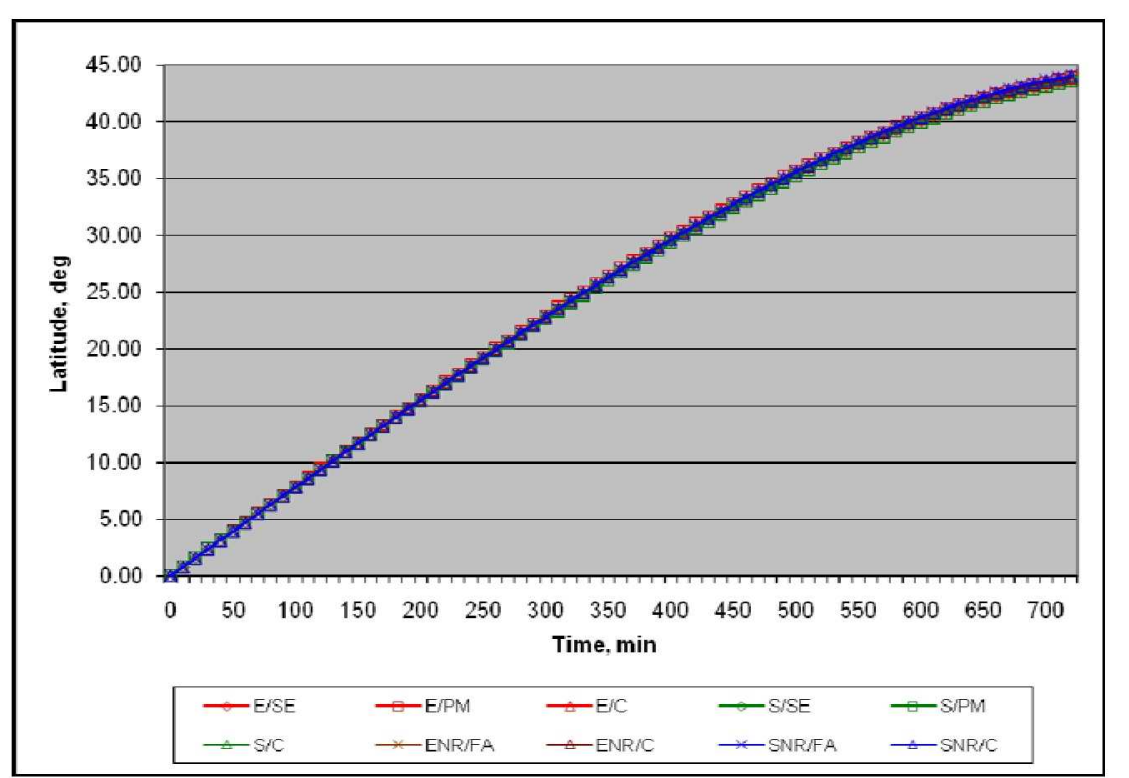

Figure 17 North-East from Equator - Latitude vs. Time

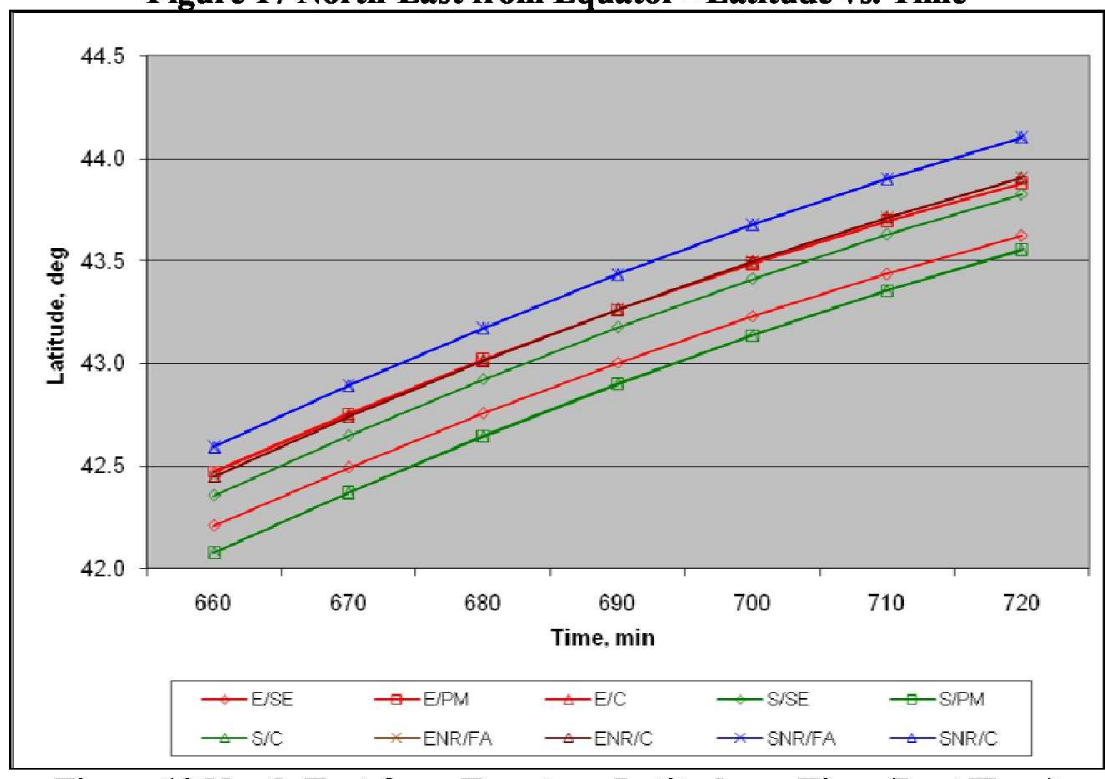

Figure 19 North-East from Equator - Latitude vs. Time (Last Hour)

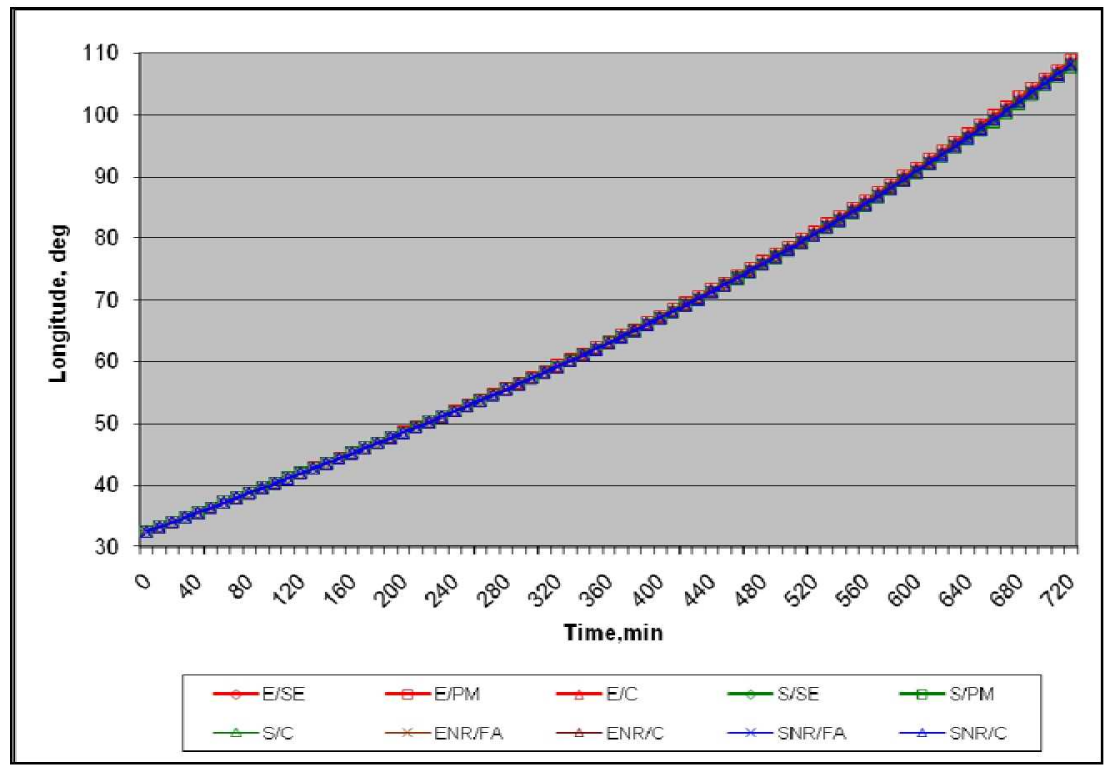

Figure 18 North-East from Equator - Longitude vs. Time

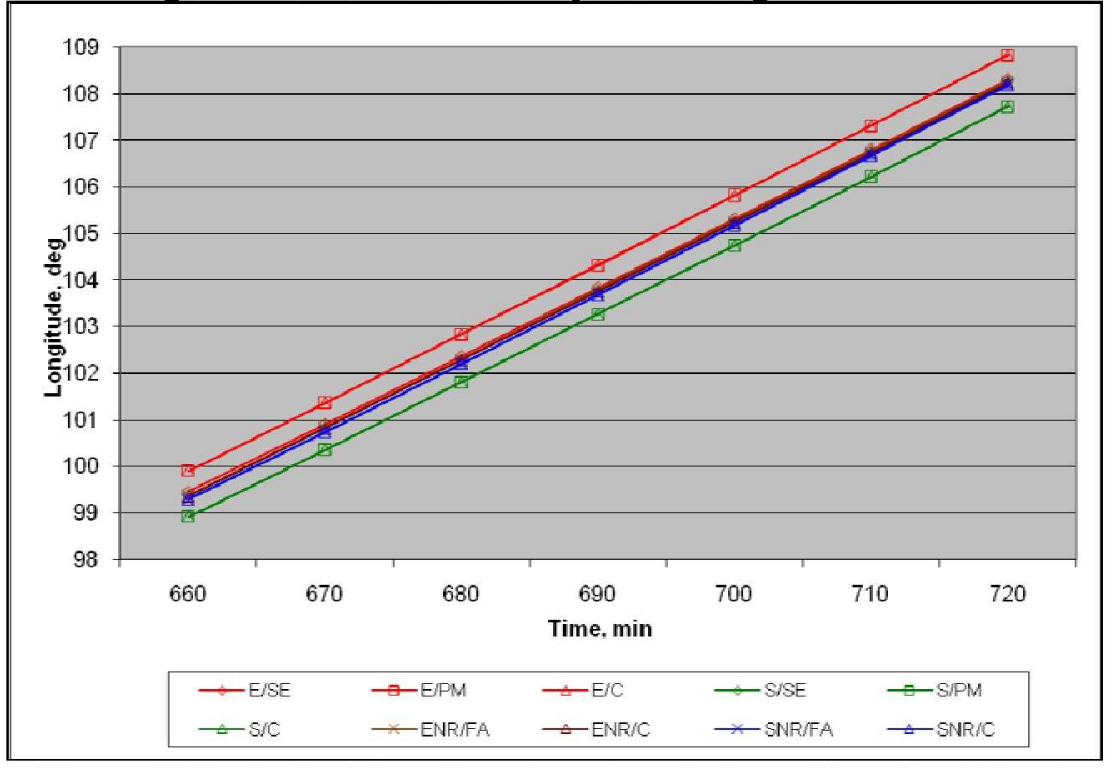

Figure 20 North-East from Equator - Longitude vs. Time (Last Hour) 


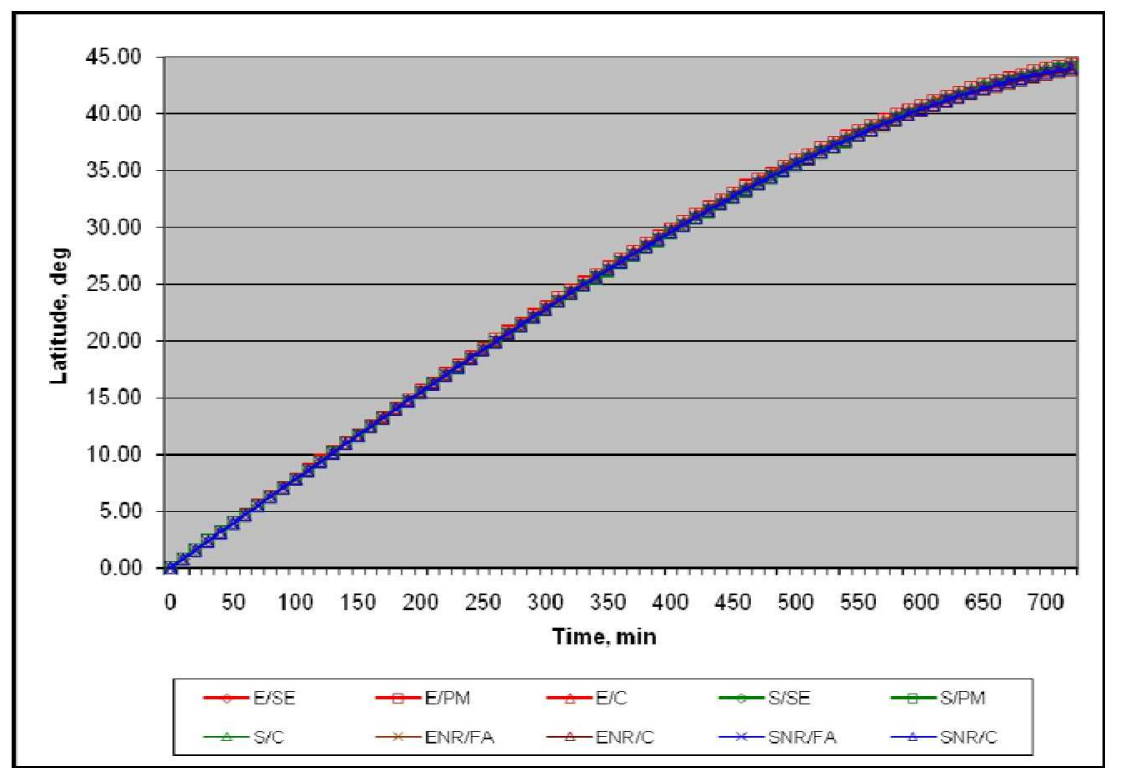

Figure 21 North-West from Equator - Latitude vs. Time

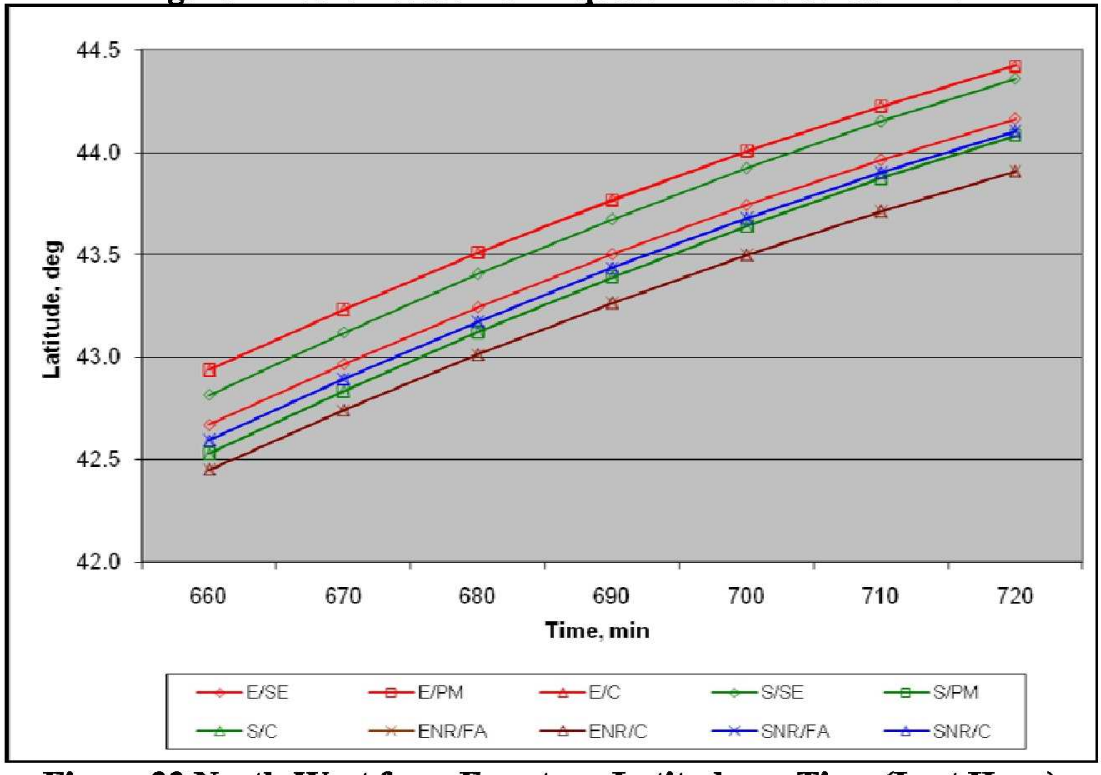

Figure 23 North-West from Equator - Latitude vs. Time (Last Hour)

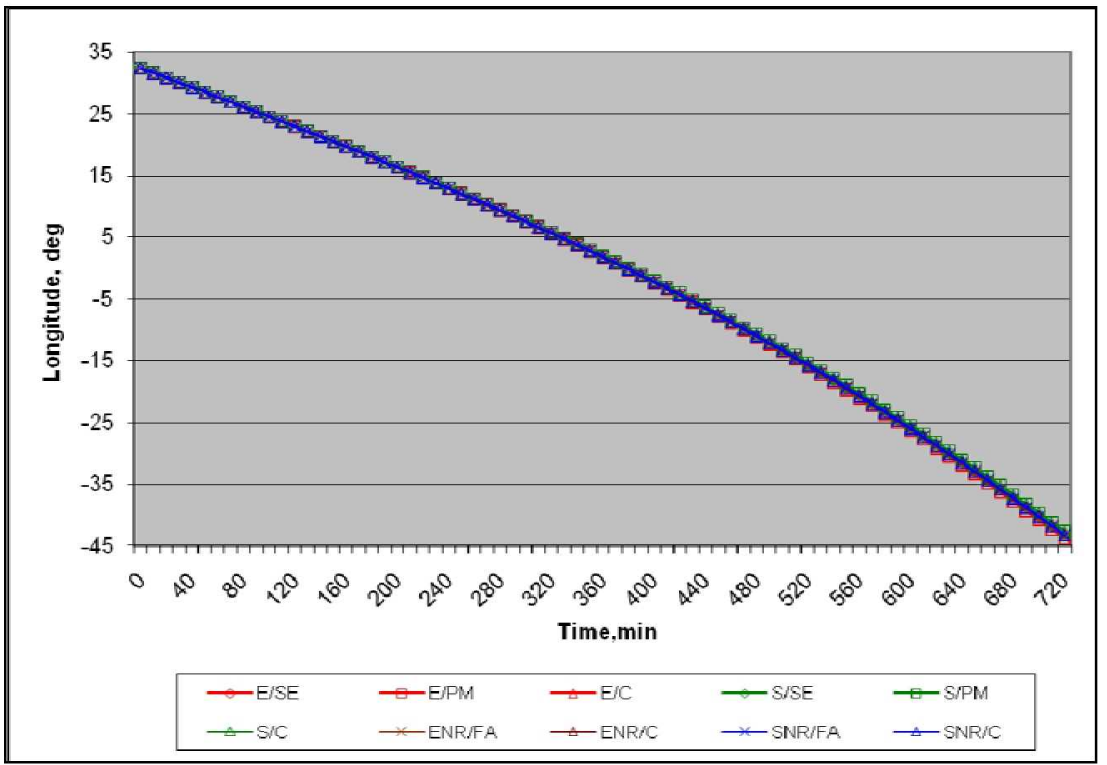

Figure 22 North-West from Equator - Latitude vs. Time

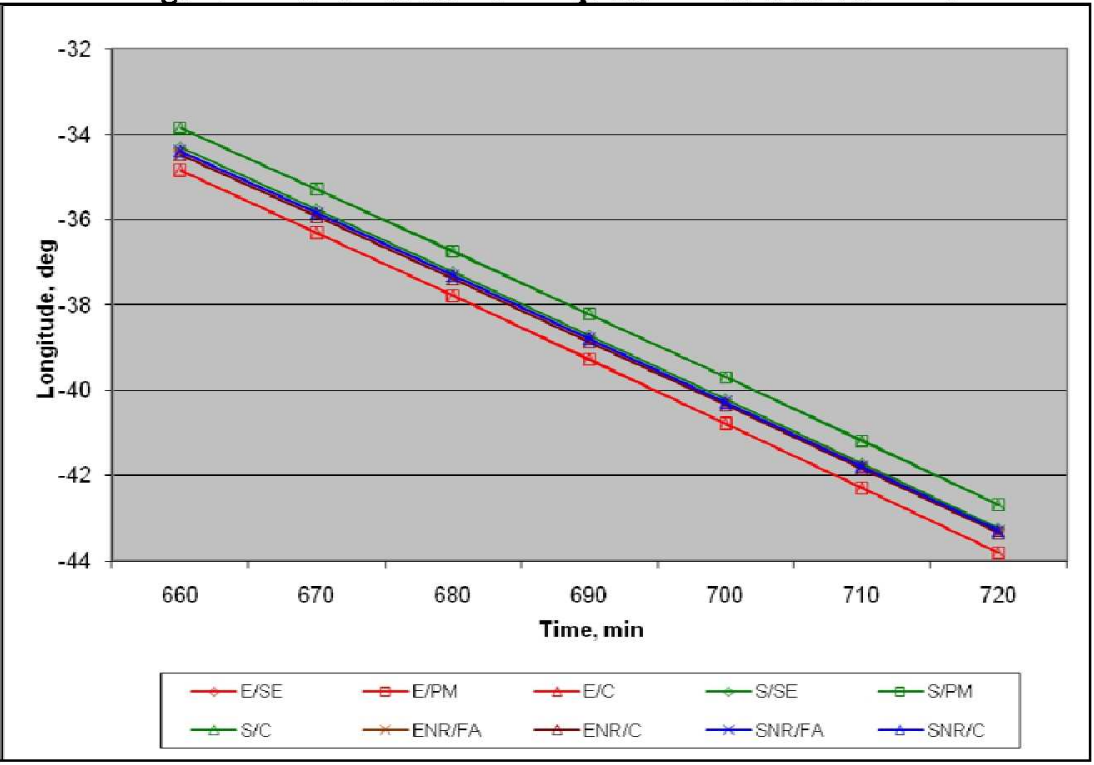

Figure 24 North-West from Equator - Longitude vs. Time (Last Hour) 


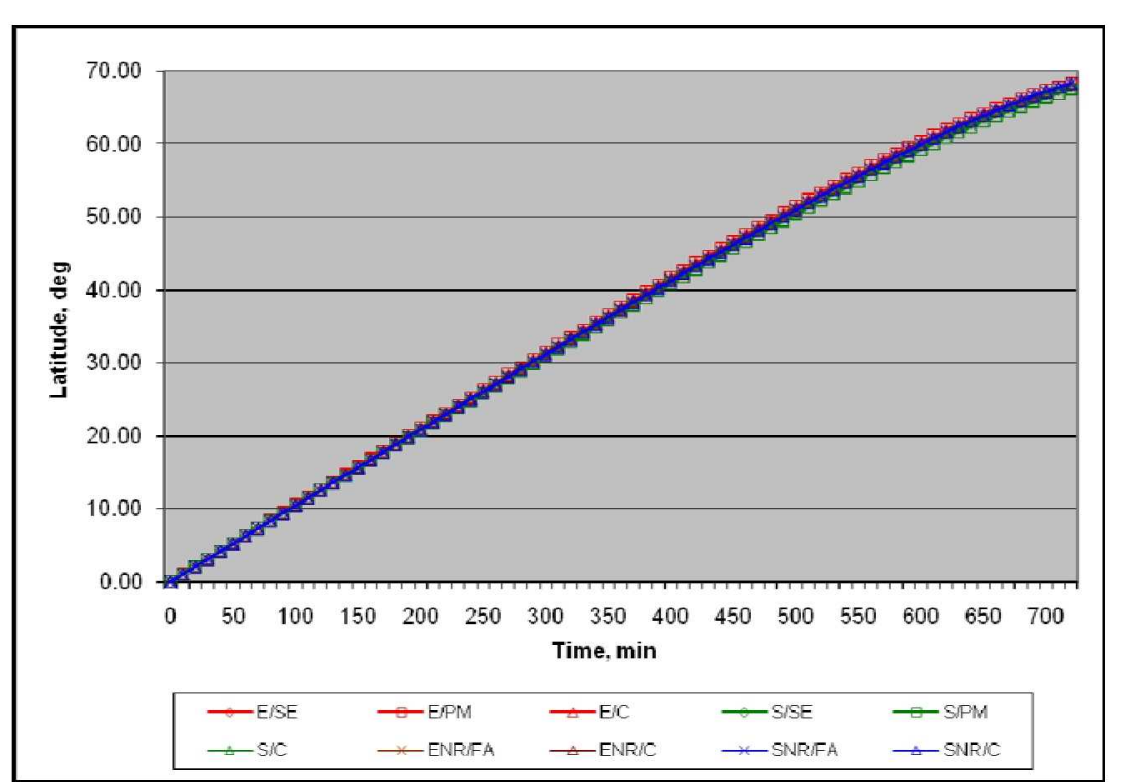

Figure $252^{\circ}$ Track from Equator - Latitude vs. Time

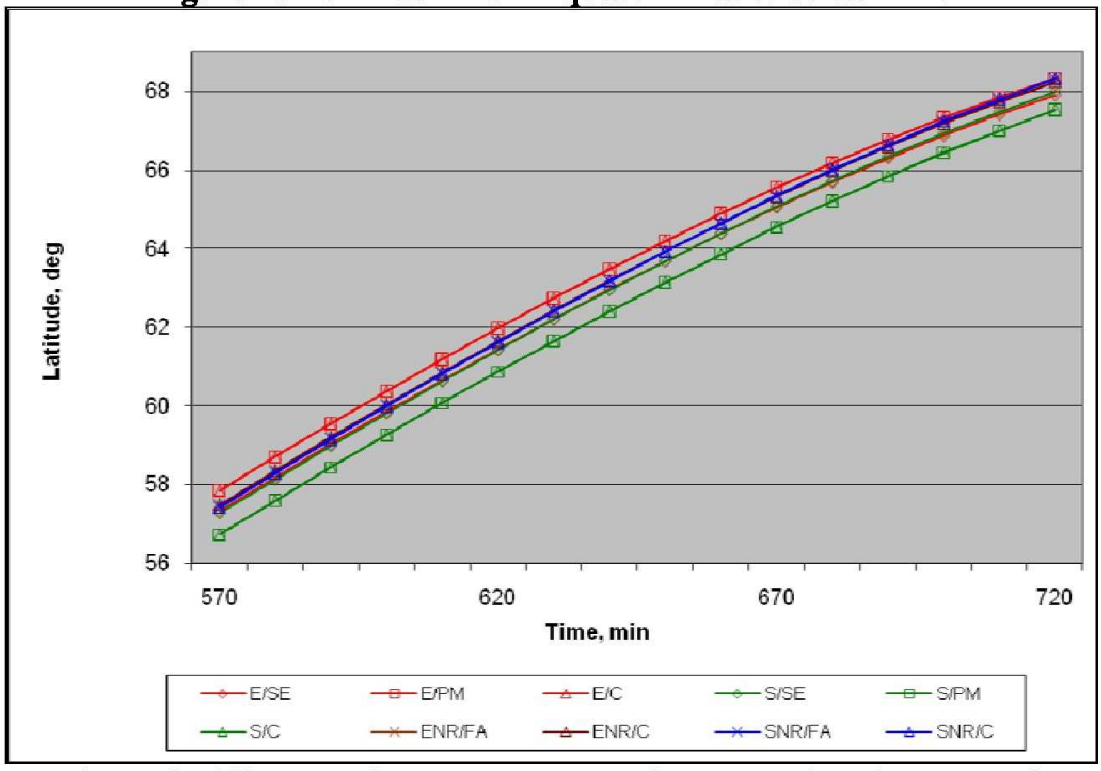

Figure $27 \mathrm{20}^{\circ}$ Track from Equator - Latitude vs. Time (Last Hour)

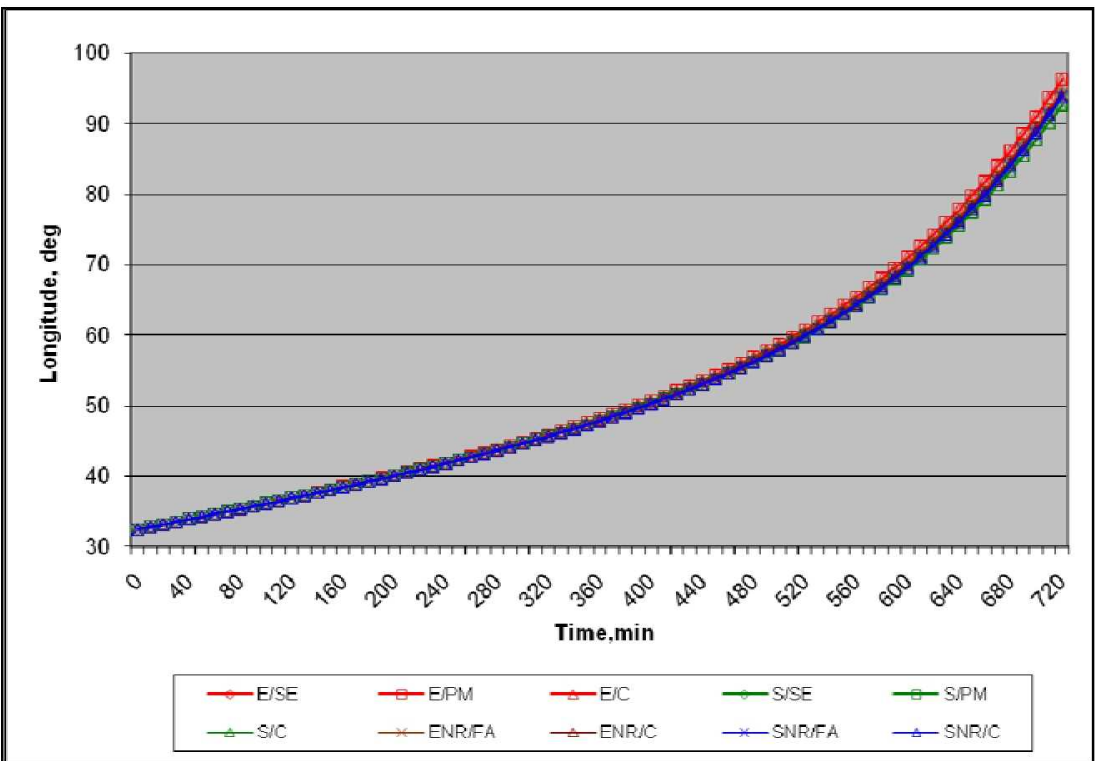

Figure $2620^{\circ}$ Track from Equator - Longitude vs. Time

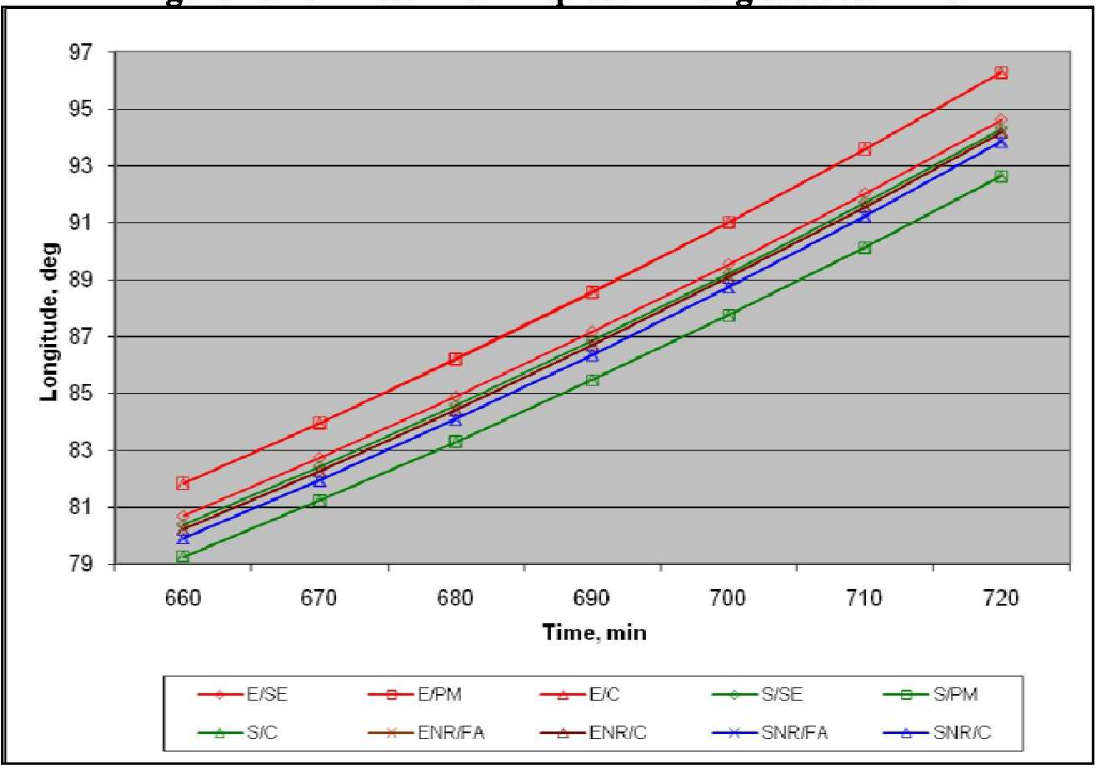

Figure $282^{\circ}$ Track from Equator - Longitude vs. Time (Last Hour) 


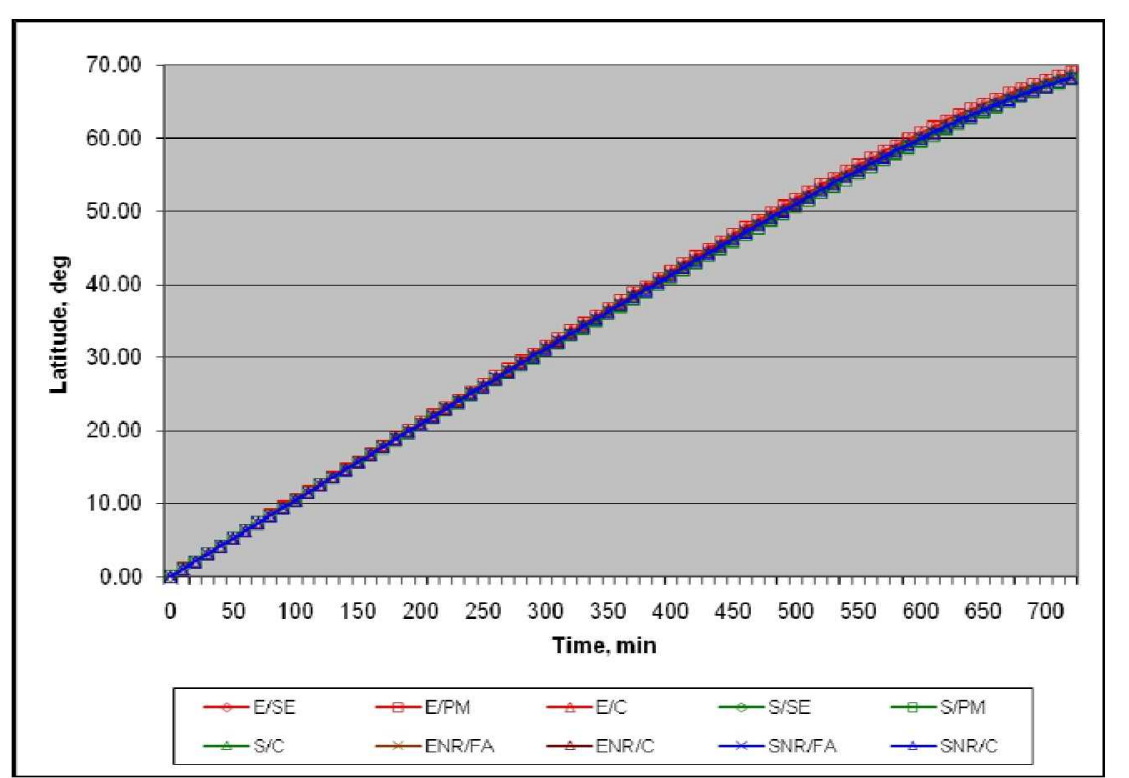

Figure $29340^{\circ}$ Track from Equator - Latitude vs. Time

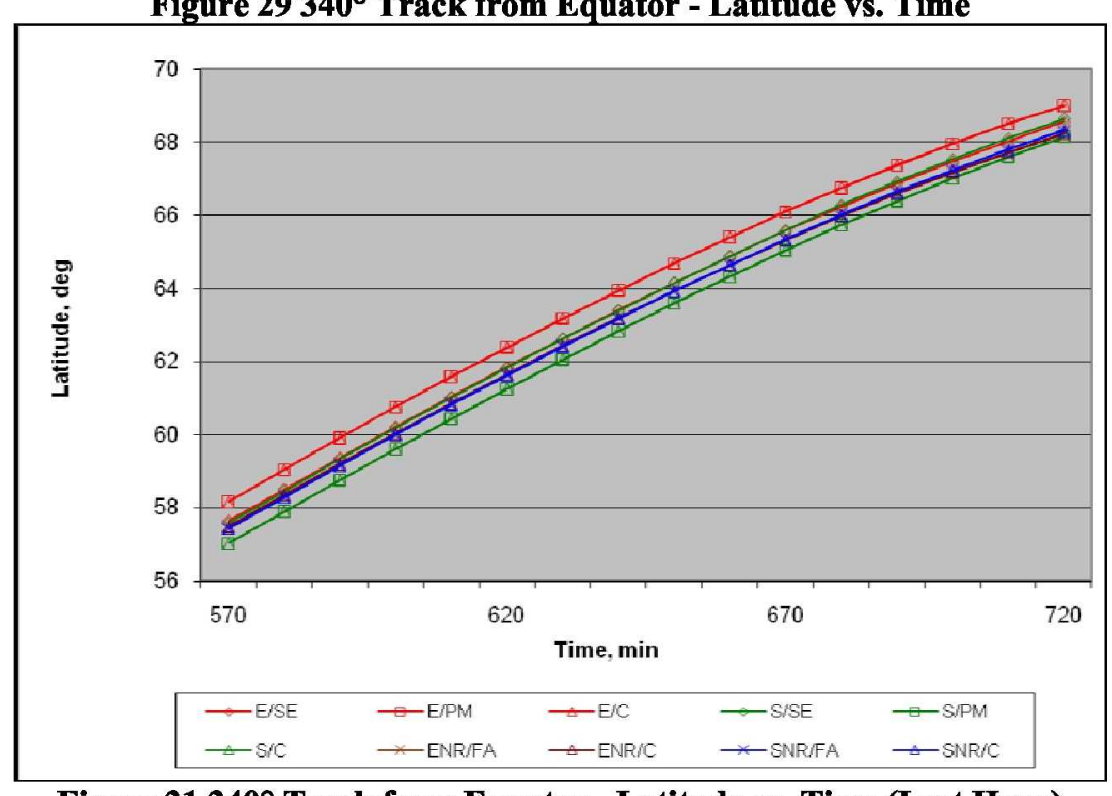

Figure $31340^{\circ}$ Track from Equator - Latitude vs. Time (Last Hour)

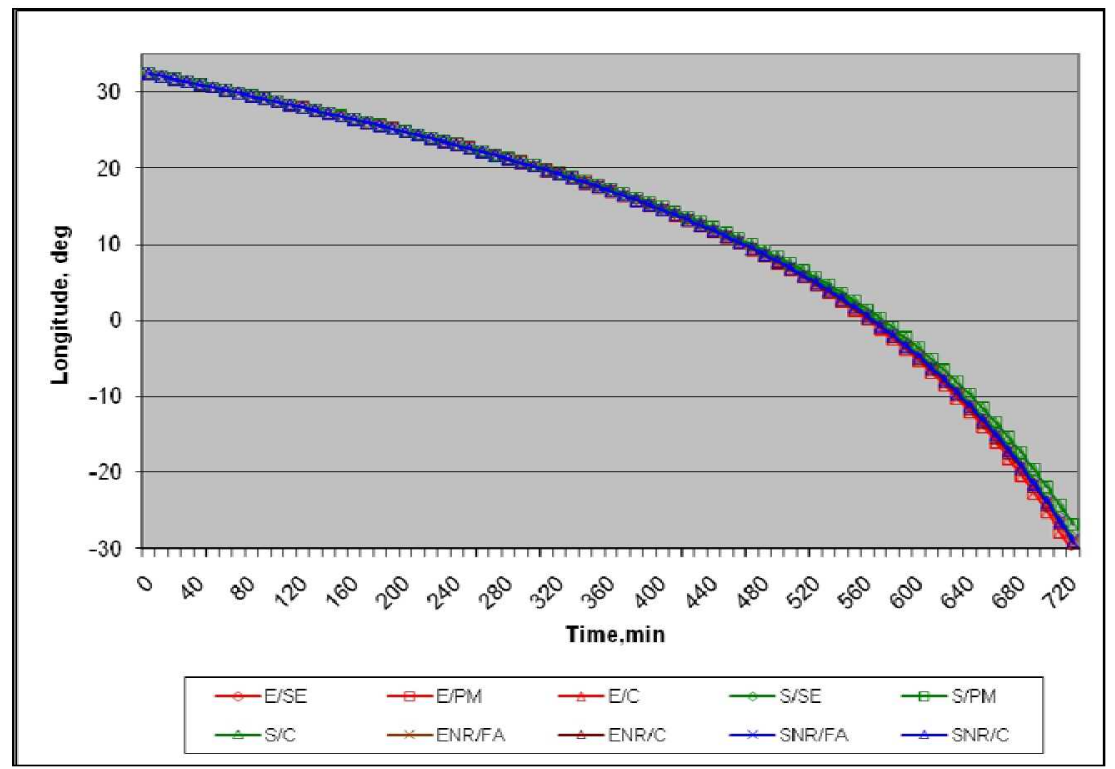

Figure $30340^{\circ}$ Track from Equator - Longitude vs. Time

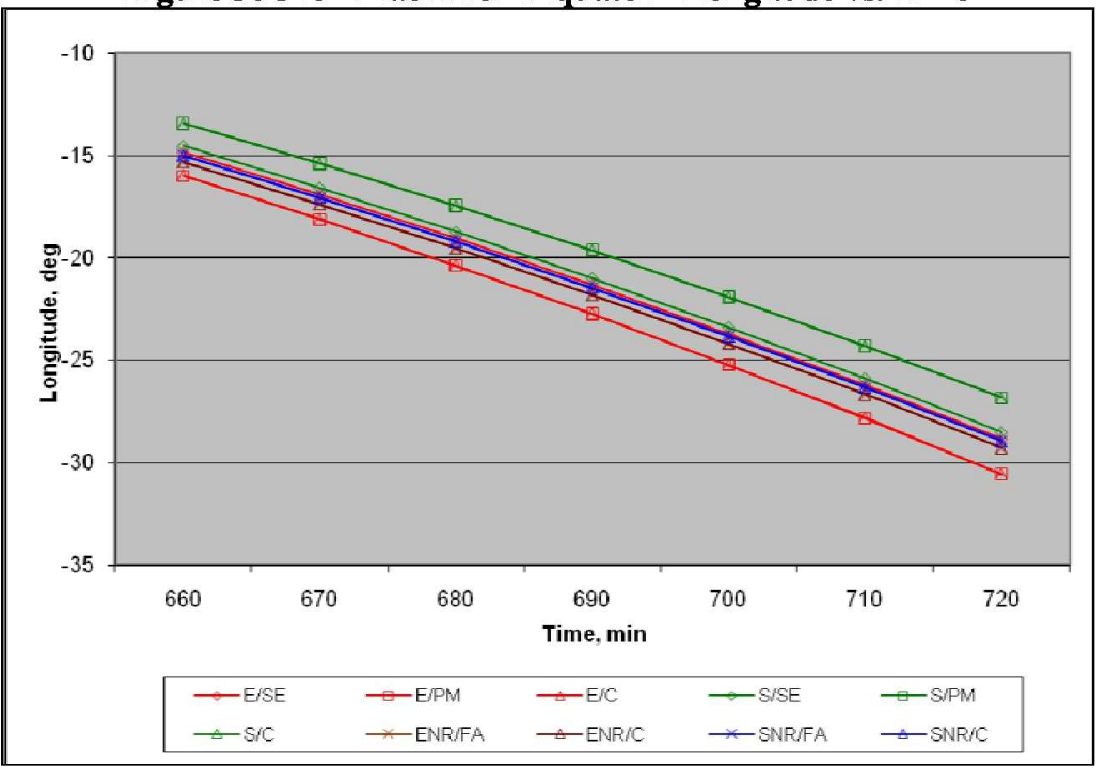

Figure $32340^{\circ}$ Track from Equator - Longitude vs. Time (Last Hour) 


\section{References}

1 R. Leslie, D. Geyer, K. Cunningham, M. Madden, P. Kenney, and P. Glaab, "LaSRS+1: An Object-Oriented Framework for Real-Time Simulation of Aircraft," AIAA Modeling and Simulation Technologies Conference and Exhibit, August 1998, Boston, Massachusetts, AIAA-98-4529.

2 Madden, Michael M. "Gravity Modeling for Variable Fidelity Environments", AIAA-2006-6730, AIAA Modeling and Simulation Technologies Conference, Keystone, CO, August 21-24, 2006.

3 "Department of Defense World Geodetic System 1984", National Imagery and Mapping Agency, NIMA TR8350.2, Third Edition, Amendment 1, 3 January 2000.

4 Madden, Michael M. "Influence of World and Gravity Model Selection on Surface Interacting Vehicle Simulations", AIAA-2007-6370, AIAA Modeling and Simulation Technologies Conference, Hilton Head, SC, August 20-23, 2007.

5 Allen, William H. (Ed.), "Dictionary of Technical Terms for Aerospace Use", National Aeronautics and Space Administration, Washington D.C., NASA SP-7, First Edition, 1965. Also available as an online database at URL: http://roland.lerc.nasa.gov/ dglover/dictionary/content.html [cited July 19, 2006].

6 Hofmann-Wellenhof, Bernhard and Moritz, Helmut, Physical Geodesy, SpringerWeinNewYork, New York, 2005, ISBN 3-211-23584-1. Sections Preface, 1.7, 2.1, 2.10.

7 Moritz, H. “Geodetic Reference System 1980", Journal of Geodesy, Vol. 74, No.1, 2000, pp. 128-133. 